\title{
Forecasting Value-at-Risk Using Nonlinear Regression Quantiles and the Intra-day Range
}

\author{
Cathy W. S. Chen ${ }^{1}{ }^{*}$ Richard Gerlach ${ }^{2}$, Bruce B. K. Hwang ${ }^{1}$, and Michael McAleer ${ }^{3}$ \\ ${ }^{1}$ Graduate Institute of Statistics and Actuarial Science, Feng Chia University, Taiwan. \\ ${ }^{2}$ University of Sydney Business School, Australia. \\ ${ }^{3}$ Erasmus School of Economics, Erasmus University Rotterdam, Netherlands, \\ Institute of Economic Research Kyoto, University Kyoto, Japan, \\ and Department of Quantitative Economics Complutense University of Madrid, Spain.
}

May 18, 2011

*Corresponding author: Cathy W.S. Chen. Fax: 88642451 7092. Email: chenws@fcu.edu.tw 


\title{
Forecasting Value-at-Risk Using Nonlinear Regression Quantiles
}

\begin{abstract}
Value-at-Risk (VaR) is commonly used for financial risk measurement. It has recently become even more important, especially during the 2008-09 global financial crisis. We propose some novel nonlinear threshold conditional autoregressive VaR (CAViaR) models that incorporate intra-day price ranges. Model estimation and inference are performed using the Bayesian approach via the link with the Skewed-Laplace distribution. We examine how a range of risk models perform during the 2008-09 financial crisis, and evaluate how the crisis affects the performance of risk models via forecasting VaR. Empirical analysis is conducted on five Asia-Pacific Economic Cooperation stock market indices as well as two exchange rate series. We examine violation rates, back-testing criteria, market risk charges and quantile loss function values to measure and assess the forecasting performance of a variety of risk models. The proposed threshold CAViaR model, incorporating range information, is shown to forecast VaR more efficiently than other models, across the series considered, which should be useful for financial practitioners.
\end{abstract}

Keywords: Value-at-Risk; CAViaR model; Skewed-Laplace distribution; intra-day range; backtesting, Markov chain Monte Carlo.

\section{Introduction}

It is well known that the bursting of the global housing bubble, especially in the USA, caused a significant reduction in real estate-based securities and a subsequent increase in the default rate of mortgages, especially sub-prime mortgages. The resulting effect on global financial markets, initially via mortgage-based assets like collateralized-debt-obligations through to bankruptcies of financial institutions and collapsing stock markets, all contributed to the global financial crisis (GFC) of 2008-09. The crisis has (once again) called into question financial risk management practices and whether risk measures can be forecast accurately enough for that purpose. This 
paper adds to this debate by proposing some novel univariate, semi-parametric range-based conditional autoregressive $\mathrm{VaR}(\mathrm{CAViaR})$ models and evaluating them for forecasting tail risk, specifically Value-at-Risk (VaR), in a "horse-race" with some existing, competing models, during the GFC period, for some individual market returns, a portfolio of these market returns, plus two exchange rate return series. The motivation is to generate more accurate and efficient forecasts of VaR for univariate asset and market returns, single fixed-weight portfolio returns and exchange rate return series, to help achieve better risk measurement and risk management practice. We attempt this by incorporating intra-day high-low price range data, known to be more efficient, at least regarding volatility estimation, than simple daily returns data since at least Parkinson (1980), into the CAViaR model. We then examine whether this adds to the efficiency and accuracy of VaR forecasts during the GFC; the evidence presented suggests this is indeed the case.

Quantitative risk measure forecasting has become very important, at least since the market crash in 1987, and even moreso after the recent global financial disaster, which began with a liquidity crisis in the U.S. banking system: the "credit-crunch"; caused by the over-valuation of assets, and included the Lehman Brothers bankruptcy, AIG crisis, and the sub-prime mortgage debacle. Financial markets and products continue to become increasingly complex, and risk management and regulations need to keep pace with this rapid process. The Basel II Accord is designed to monitor and encourage sensible risk taking, using appropriate models to calculate $\mathrm{VaR}$ and daily capital charges. VaR is now a standard tool in risk management and became highly important following the 1995 amendment to the Basel Accord, whereby banks and other Authorized Deposit-taking Institutions (ADIs) were permitted to use internal models to forecast daily VaR. VaR was pioneered by J.P. Morgan Corporation, via their RiskMetrics system, in 1993 and is more formally defined by Jorion (1996), as an estimate of the probability and size of the worst potential or expected loss over a given time horizon with a specified probability. Mathematically:

$$
P_{r}\left(\Delta V(l) \leq-\operatorname{VaR} \mid \mathcal{F}_{t-1}\right)=\alpha,
$$

where $\Delta V(l)$ is the change in the asset value over time period $l, \alpha$ is the probability level, and $\mathcal{F}_{t-1}$ denotes the information set at time $t-1$. Models and methods for VaR forecasting are an on-going challenge for financial practitioners and statisticians.

In this paper, we propose a new semi-parametric family of quantile risk CAViaR models; discuss the selection of optimal risk models; examine how risk management strategies performed 
during the 2008-09 GFC; evaluate how the crisis affected risk management practices, forecasts of VaR and daily capital charges; and discuss diagnostic checking of VaR methods. Further, we adapt the Bayesian estimation methods in Yu and Moyeed (2001), exploiting the link between quantile estimation and the Skewed-Laplace distribution, first discussed by Koenker and Machado (1999), to the range of models in the CAViaR family in a systematic way, conducting a comparison with the frequentist estimation of Engle and Manganelli (2004), in regards to the forecasts produced from these models.

The paper is structured as follows. In Section 2, methods for VaR are reviewed and the new CAViaR specifications, incorporating range information, are presented. Section 3 discusses estimation of VaR models and criteria for measuring VaR performance. Empirical analysis is conducted in Section 4 on five Asia-Pacific Economic Cooperation (APEC) stock market indices, including Standard and Poors 500 Index, Nikkei 225, TAIEX, HSI and KOSPI, to forecast VaR from August 2008 to April 2010. Finally, some concluding remarks are given in Section 5.

\section{Value-at-Risk - Models and Methods}

For a given value $\alpha, 0 \leq \alpha \leq 1$, the $\alpha$ th quantile of the variable $y$ is defined as $q_{\alpha}(y)=$ $\inf \{y \mid F(y) \geq \alpha\}$, where $F$ is the CDF of $y$. There are several VaR estimation methods in the literature, classified into three over-arching categories:

Non-parametric: no, or very few or non-restrictive, assumptions are made on the distribution of returns, e.g. historical simulation, which uses past sample return quantiles.

Parametric: usually constructed assuming a specific choice for the unconditional and/or conditional return distribution and also the model dynamics. Kuester et al. (2006) conduct a review of some competing models, many with generalized autoregressive conditional heteroscedastic (GARCH) volatility equations (proposed by Engle, 1982, and Bollerslev, 1986) with specific noise distributions such as Gaussian, Student-t, skewed Student-t (see Hansen, 1994). McAleer and da Veiga (2008a) propose a parsimonious portfolio spill-over GARCH (PS-GARCH) model which accommodates aggregate spill-overs, and avoids the so-called curse of dimensionality. Chen et al. (2011) also consider a range of parametric models to forecast VaR, including standard, threshold nonlinear and Markov switching GARCH specifications (see e.g. Guidolin and Timmerman, 2006; or Haas, Mittnik and Paolella, 2006), plus standard and nonlinear stochastic volatility models, together with four probability distributions for the error component, namely Gaussian, Student-t, skewed Student-t, and generalized error distributions. 
Semi-parametric: often makes assumptions about the model dynamics but not the error distribution, e.g. Engle and Manganelli (2004) propose direct dynamic quantile regression (see Koenker and Bassett, 1978) to calculate VaR, denoted CAViaR, which directly models the dynamics of each quantile. Gerlach, Chen and Chan (2011) propose a family of nonlinear CAViaR models, extended from those in Engle and Manganelli (2004).

In this paper we use methods from all three classes above. Historical simulation is employed in the non-parametric category, where we use two sample percentiles: a short-term (ST, the last 25 days) and a long-term (LT, last 100 days).

For parametric methods, RiskMetrics and GARCH models are used. More precisely, the IGARCH $(1,1)$ of RiskMetrics with Gaussian errors, and the $\operatorname{GARCH}(1,1)$ model with Gaussian and Student-t errors, are considered in the empirical analysis. Much of the literature on VaR forecasting focuses on these models as benchmarks. The models are specified as follows:

Model A: GARCH model

$$
\begin{aligned}
& y_{t} \quad=\mu_{t}+a_{t}, \mu_{t}=\phi_{0}+\phi_{1} y_{t-1}, \\
& a_{t} \quad=\varepsilon_{t} \sqrt{h_{t}}, \text { where } \varepsilon_{t} \stackrel{i . i . d}{\sim} D(0,1), \\
& h_{t} \quad=\alpha_{0}+\alpha_{1} a_{t-1}^{2}+\beta_{1} h_{t-1} .
\end{aligned}
$$

Model B: RiskMetrics model

$$
\begin{aligned}
& y_{t} \quad=a_{t} \\
& a_{t} \quad=\varepsilon_{t} \sqrt{h_{t}}, \text { where } \varepsilon_{t} \stackrel{i . i . d}{\sim} N(0,1) \\
& h_{t} \quad=(1-\lambda) a_{t-1}^{2}+\lambda h_{t-1} .
\end{aligned}
$$

Under each model, the one-step-ahead VaR at $\alpha \%$ quantile level is computed, as $V a R_{t}=$ $\mu_{t}+D_{\alpha}^{-1} \sqrt{h_{t}}$, where $D^{-1}$ is the inverse CDF for the distribution $D$. The parameters of the GARCH models are estimated by Bayesian Markov chain Monte Carlo (MCMC), as in Chen, Chiang and So (2003) and discussed in the next section.

For semi-parametric models we consider the CAViaR models discussed in the next section. Giacomini and Komunjer (2005) find that CAViaR is most efficient at the $1 \%$ quantile level, but the GARCH model with normal distributed errors is better than CAViaR at the $5 \%$ quantile level. Gerlach et al (2011) find similar results across a range of financial market indices. McAleer et al. (2010a) consider mixing alternative risk models, and discuss the choice between conservative and aggressive risk management, as well as evaluating the effects of the Basel II 
Accord for risk management. McAleer et al. (2010b) provide a method for choosing one risk model at the beginning of the period, and then modify the forecast depending on the recent history of violations.

\subsection{Quantile regression and CAViaR}

Koenker and Bassett (1978) suggest that, based on a sample of i.i.d. realizations $\left\{y_{t}\right\}$ of $y$, the quantile $b=q_{\alpha}(y)$ can be estimated by solving the following minimization problem:

$$
\min _{b \in \Re}\left[\sum_{t}\left(y_{t}-b\right)\left(\alpha-I\left\{y_{t}<b\right\}\right)\right] .
$$

Engle and Manganelli (2004) propose some time series models for the quantile, i.e. $b$ becomes $b_{t}$ and the i.i.d. assumption is relaxed, called CAViaR, and apply this criterion to estimate the unknown parameters in the models for $b_{t}$. Let $y_{t}$ be an asset, market, portfolio or exchange rate return at time $t$, and $\boldsymbol{\beta}_{\alpha}$ the vector of $q+r$ unknown parameters, $\left(\beta_{1}, \ldots, \beta_{q}, \beta_{q+1}, \ldots, \beta_{q+r}\right)^{\prime}$, for the $\alpha$-quantile model. For notational convenience, we let $f_{t}(\boldsymbol{\beta})=f_{t}\left(y_{t}, \boldsymbol{\beta}_{\alpha}\right)$ denote the time $t$ conditional $\alpha$ level quantile. A general specification of $\mathrm{VaR}$ at time $t$ is:

$$
f_{t}(\boldsymbol{\beta})=\beta_{0}+\sum_{i=1}^{q} \beta_{i} f_{t-i}(\boldsymbol{\beta})+g\left(\beta_{q+1}, \ldots, \beta_{q+r}, \mathcal{F}_{t-1}\right),
$$

where $g()$ is a function of a finite number of lagged returns and model parameters, thus linking the alpha quantile $f_{t}(\boldsymbol{\beta})$ to past returns, which are a subset of all past information, denoted as $\mathcal{F}_{t-1} . \quad \beta_{i} f_{t-i}(\boldsymbol{\beta})$ is the autoregressive term which ensures smooth quantile changes over time. Three general CAViaR specifications in Engle and Manganelli (2004) are:

(1) Symmetric Absolute Value (SAV):

$$
f_{t}(\boldsymbol{\beta})=\beta_{1}+\beta_{2} f_{t-1}(\boldsymbol{\beta})+\beta_{3}\left|y_{t-1}\right|
$$

(2) Asymmetric Slope (AS):

$$
f_{t}(\boldsymbol{\beta})=\beta_{1}+\beta_{2} f_{t-1}(\boldsymbol{\beta})+\left(\beta_{3} I_{\left(y_{t-1}>0\right)}+\beta_{4} I_{\left(y_{t-1}<0\right)}\right)\left|y_{t-1}\right| .
$$

(3) Indirect GARCH(1,1) (IG):

$$
f_{t}(\boldsymbol{\beta})=\left(\beta_{1}+\beta_{2} f_{t-1}^{2}(\boldsymbol{\beta})+\beta_{3} y_{t-1}^{2}\right)^{1 / 2}
$$


Yu, Li, and Jin (2011) extend CAViaR using two approaches, namely the threshold and mixture type indirect-GARCH CAViaR models. Gerlach et al (2011) propose a nonlinear CAViaR model to capture more flexible asymmetric and nonlinear responses via more general threshold nonlinear forms. We adopt the threshold CAViaR (TCAV) model of Gerlach et al (2011), and threshold-type indirect-VaR model of $\mathrm{Yu}, \mathrm{Li}$, and Jin (2011) as follows:

(4) Threshold CAViaR (TCAV)

$$
f_{t}(\boldsymbol{\beta})= \begin{cases}\beta_{1}+\beta_{2} f_{t-1}(\boldsymbol{\beta})+\beta_{3}\left|y_{t-1}\right|, & z_{t-1} \leq \gamma \\ \beta_{4}+\beta_{5} f_{t-1}(\boldsymbol{\beta})+\beta_{6}\left|y_{t-1}\right|, & z_{t-1}>\gamma\end{cases}
$$

where $\boldsymbol{z}$ is an observed threshold variable, which can be exogenous or self-exciting (i.e. $z_{t}=y_{t}$ ), and $\gamma$ is the threshold value, typically set as $\gamma=0$. We extend the model slightly by estimating this parameter in this paper, while Gerlach et al (2011) fix $\gamma=0$. Further:

(5) Threshold Indirect GARCH(1,1) (TIG):

$$
f_{t}(\boldsymbol{\beta})= \begin{cases}\left(\beta_{1}+\beta_{2} f_{t-1}^{2}(\boldsymbol{\beta})+\beta_{3} y_{t-1}^{2}\right)^{1 / 2}, & \text { if } y_{t-1}<\gamma, \\ \left(\beta_{4}+\beta_{5} f_{t-1}^{2}(\boldsymbol{\beta})+\beta_{6} y_{t-1}^{2}\right)^{1 / 2}, & \text { if } y_{t-1} \geq \gamma .\end{cases}
$$

\subsection{Proposed Range-based CAViaR models}

There are several advantages to using the intra-day high-low price range directly for volatility measurement and forecasting, relative to the use of absolute or squared return data, or intraday returns. Many papers have shown the intra-day range to be an efficient measure of daily volatility (e.g. see Parkinson, 1980). Mandelbrot (1971) proposes the range to evaluate the existence of long-term dependence on asset prices; Garman and Klass (1980) show that high-low price-range data contain more information regarding volatility than opening to closing prices. Beckers (1983) applies the range estimator to incorporate past information for different variance measures. Gallant et al. (1999) and Alizadeh, Brandt and Diebold (2002) incorporate the range into the stochastic volatility model. Brandt and Jones (2006) proposed a range-based EGARCH model, using a link between the range and intra-day volatility, showing that the their model had favourable out-of-sample volatility forecasting performance. Chou (2005) proposes the Conditional Autoregressive Range (CARR) model for the high and low range of asset prices. Chen, Gerlach and Lin (2008) allow the intra-day high and low price range to depend nonlinearly on past information, or an exogenous variable such as US market information, finding increased accuracy for volatility estimation over the CARR and GARCH models. Here, we propose a family of CAViaR models that incorporates intra-day price range information. 
In the same spirit as Chou (2005) and Chen et al (2008), we extend the CAViaR models in (2), (4), (5) and incorporate the intra-day high-low price range into the following models:

(6) Range Value (RV):

$$
f_{t}(\boldsymbol{\beta})=\beta_{1}+\beta_{2} f_{t-1}(\boldsymbol{\beta})+\beta_{3} R_{t-1}
$$

(7) Threshold Range Value (TRV):

$$
f_{t}(\boldsymbol{\beta})= \begin{cases}\beta_{1}+\beta_{2} f_{t-1}(\boldsymbol{\beta})+\beta_{3} R_{t-1}, & \text { if } R_{t-1} \leq \gamma, \\ \beta_{4}+\beta_{5} f_{t-1}(\boldsymbol{\beta})+\beta_{6} R_{t-1}, & \text { if } R_{t-1}>\gamma\end{cases}
$$

The first model has the same form as the SAV model in (1), but replaces the absolute return with the intra-day price range $R_{t-1}$. The TRV has the same form as the TCAV in (4), again replacing return data with range data. The following model makes the same adjustments to the TIG model in (5):

(8) Threshold Range Indirect GARCH(1,1) (TRIG):

$$
f_{t}(\boldsymbol{\beta})= \begin{cases}\left(\beta_{1}+\beta_{2} f_{t-1}^{2}(\boldsymbol{\beta})+\beta_{3} R_{t-1}^{2}\right)^{1 / 2}, & \text { if } R_{t-1} \leq \gamma, \\ \left(\beta_{4}+\beta_{5} f_{t-1}^{2}(\boldsymbol{\beta})+\beta_{6} R_{t-1}^{2}\right)^{1 / 2}, & \text { if } R_{t-1}>\gamma\end{cases}
$$

Here $R_{t}$ is the intra-day range at time $t$, and $\gamma$ is the threshold value. The RV model responds symmetrically to past range, while the TRV and TRIG allow for different responses to high and low ranges.

\section{Estimation and Forecast Evaluation}

Using the Koenker and Bassett (1978) regression quantile framework, the unknown parameters of CAViaR models can be estimated by optimising a criterion function. The $\alpha$ th regression quantile is defined as the solution, $\boldsymbol{\beta}_{\alpha}$, of the criterion function:

$$
\min \sum\left(y_{t}-f_{t}(\boldsymbol{\beta})\right)\left\{\alpha-I_{(-\infty, 0)}\left(y_{t}-f_{t}(\boldsymbol{\beta})\right)\right\}
$$

where $f_{t}(\boldsymbol{\beta})$ is the model for the $\alpha$ th regression quantile. Based on a sample of data $y_{1}, \ldots, y_{n}$, the function (9) can be numerically minimised to find $\hat{\boldsymbol{\beta}}_{\alpha}$, as was done by Engle and Manganelli (2004) for CAViaR models (1)-(3). Chen et al (2011) also use this method for estimation in the 
TCAV in (4), then show that for simulated data, the Bayesian estimate using MCMC is more efficient for that model. We discuss this approach now.

It has recently been shown that the quantile regression criterion function is related to the likelihood function for the skewed-Laplace distribution. This result allows (maximum) likelihood estimation, and has motivated Bayesian solutions for this problem, as proposed in $\mathrm{Yu}$ and Moyeed (2001), Tsionas (2003), Yu and Zhang (2005) and Geraci and Bottai (2007), and subsequently extended in Chen et al (2011). These designs all involve MCMC computational methods due to the non-standard form of the posterior resulting from the skewed-Laplace likelihood.

\subsection{Frequentist estimation}

First, we define the data vectors as $\boldsymbol{y}=\left(y_{1}, \ldots, y_{n}\right)^{\prime}$ for the asset returns and $\boldsymbol{R}=\left(R_{1}, \ldots, R_{n}\right)^{\prime}$ for the intra-day range data. If we assume the returns follow a skewed-Laplace, i.e. $y_{t} \stackrel{i . i . d}{\sim}$ $S L\left(f_{t}(\boldsymbol{\beta}), \tau, \alpha\right)$, then, the following density function results:

$$
f\left(y_{t} ; f_{t}(\boldsymbol{\beta}), \tau, \alpha\right)=\frac{\alpha(1-\alpha)}{\tau} \exp \left[-\rho_{\alpha}\left(\frac{y_{t}-f_{t}(\boldsymbol{\beta})}{\tau}\right)\right],
$$

where $\rho_{\alpha}(u)=u(\alpha-I(u<0)), f_{t}(\boldsymbol{\beta})$ is the mode and $\tau>0$ is a scale parameter. Under this assumption, the likelihood function for any CAViaR model, including (1) -(8), is then:

$$
L_{\alpha}(\boldsymbol{\beta}, \tau, \gamma ; \boldsymbol{y}, \boldsymbol{R}) \propto \tau^{-n} \exp \left\{-\tau^{-1}\left[\sum_{t=1}^{n}\left(y_{t}-f_{t}(\boldsymbol{\beta})\right)\left(\alpha-I_{(-\infty, 0)}\left(y_{t}-f_{t}(\boldsymbol{\beta})\right)\right)\right]\right\} .
$$

As such, the $\hat{\boldsymbol{\beta}}_{\alpha}$ that minimises (9) also maximises (10). This estimate can then simply be plugged into the formula for $f_{n+1}(\boldsymbol{\beta})$ to forecast VaR.

\subsection{Bayesian estimation and forecasting}

Bayesian inference requires specifying a prior distribution for the unknown parameters, combined with the likelihood function. Assuming the parameters, $(\boldsymbol{\beta}, \tau, \gamma)$, are a priori independent, we choose $\pi(\tau) \propto \tau^{-1}$, the standard Jeffreys' prior, and $\pi(\boldsymbol{\beta}) \propto 1$, as in Gerlach et al (2011). When considering two regimes, a flat prior on the threshold limit $\gamma$ is $\operatorname{Unif}(u, l)$, where the $(u, l)$ are chosen as suitable quantiles of the threshold variable to allow reasonable sample size in each regime for inference.

MCMC methods sample from the joint posterior distribution of the unknown model parameters for estimation, inference and forecasting. Here groups of parameters are defined for the following sampling scheme:

$$
p(\boldsymbol{\beta} \mid \boldsymbol{y}, \boldsymbol{R}, \gamma), p(\tau \mid \boldsymbol{y}, \boldsymbol{R}, \boldsymbol{\beta}, \gamma), \text { and } p(\gamma \mid \boldsymbol{y}, \boldsymbol{R}, \boldsymbol{\beta}, \tau)
$$


which is iteratively sampled from to form a dependent sample from the joint posterior distribution. The density $p(\tau \mid \boldsymbol{y}, \boldsymbol{R}, \boldsymbol{\beta}, \gamma)$ is an inverse gamma distribution, allowing $\tau$ to be integrated out of the full posterior to obtain the marginal posterior distribution $p(\boldsymbol{\beta} \mid \boldsymbol{y}, \boldsymbol{R}, \gamma)$. As all parameters have non-standard posterior densities, we use the Metropolis-Hastings (MH) algorithms (Metropolis et al., 1953, and Hastings, 1970).

In order to speed convergence and allow optimal mixing properties, we use the combined Random Walk and Independent Kernel MH algorithms. The Random Walk Metropolis algorithm is used for the first $M$ iterations, the so-called burn-in period, while the Independent Kernel $\mathrm{MH}$ algorithm is employed from iteration $M+1$ onwards, employing the sample mean and covariance matrix of the burn-in iterates for each parameter grouping. This procedure is discussed in detail in Chen and So (2006).

Bayesian forecasts of VaR can be constructed via the MCMC sampling scheme. For each MCMC iterate of parameter values $\boldsymbol{\beta}^{(j)}, \gamma^{(j)}, j=1, \ldots, M$, a 1-day $\alpha$-level VaR estimator is obtained by plugging in $\boldsymbol{\beta}^{(j)}, \gamma^{(j)}$ to the formula for $f_{n+1}(\boldsymbol{\beta})$, obtaining $f_{n+1}(\boldsymbol{\beta})^{(j)}$. Under the TRV model, this is:

$f_{n+1}(\boldsymbol{\beta})^{(j)}=\left(\beta_{1}^{(j)}+\beta_{2}^{(j)} f_{n}(\boldsymbol{\beta})+\beta_{3}^{(j)} R_{n}\right) I\left(R_{n} \leq \gamma^{(j)}\right)+\left(\beta_{4}^{(j)}+\beta_{5}^{(j)} f_{n}(\boldsymbol{\beta})+\beta_{6}^{(j)} R_{n}\right)\left(1-I\left(R_{n} \leq \gamma^{(j)}\right)\right)$.

These iterated values $f_{n+1}(\boldsymbol{\beta})^{(j)}$ are then simply averaged over the iterates $j=M+1, \ldots, N$, to obtain a posterior mean estimate $\widehat{V a R}_{n+1}$ that is a forecast of VaR, where the parameters have been integrated out in the MCMC sampling scheme.

\subsection{Parametric GARCH estimation}

The parametric GARCH models, labelled Model A above, are here estimated by MCMC methods, following the method in Chen, Chiang and So (2003). First, the standard prior choices are made, so that the the usual stationarity and positivity conditions are enforced, i.e.:

$$
\alpha_{0}>0 ; 0<\alpha_{1}+\beta_{1}<1 ; \alpha_{1}, \beta_{1} \geq 0 ;\left|\phi_{1}\right|<1
$$

and the degrees of freedom for the Student-t errors is restricted to be above 4, ensuring its' first four moments are finite. These are achieved by placing a flat prior over the parameters constrained to the region $B$, which is equivalent to (11), and defining $\eta^{*}=1 / \eta$ and using a flat prior $\eta^{*} \sim \operatorname{Unif}(0,0.25)$. Thus, the prior becomes:

$$
p\left(\phi_{1}, \phi_{1}, \alpha_{0}, \alpha_{1}, \beta_{1}\right)=I(B) \times I(\eta>4) .
$$

The likelihood is defined by the choice of error distribution, combined with the GARCH volatility equation. 
Multiplying the likelihood and the prior gives the posterior density function (up to a proportionality constant). The standard Gaussian random walk Metropolis method is employed for the first M MCMC iterations ( $\mathrm{M}$ is the size of the burn-in sample) for each of the parameter groups: (i) $\left(\phi_{1}, \phi_{1}\right)$;(ii) $\left(\alpha_{0}, \alpha_{1}, \beta_{1}\right)$; and (iii) $\eta^{*}$; in turn. After the burn-in period, the sample mean and sample variance-covariance of the iterates for $\left(\alpha_{0}, \alpha_{1}, \beta_{1}\right)$ are collected. These are then used as the proposal mean and covariance matrix in an independent kernel Metropolis-Hastings method, with a Gaussian proposal distribution. The overall method is thus adaptive, because it learns from the burn-in period. This has the added advantage of capturing the posterior correlations among the $\boldsymbol{\alpha}$ in the burn-in period for use in the sample period proposal, which should also increase efficiency. In particular, since the burn-in sample's mean (now the proposal mean) is likely not too close to the boundaries in (11), the sampler should be more efficient in that region for these parameters. For more details of this method, see Gerlach and Chen (2008) or Chen, Chiang and So (2003).

We note that this method will only work if the MCMC sample has converged and sufficiently covered the posterior inside the burn-in period. Convergence is thus monitored heavily using trace and ACF plots, while the tuning algorithm will also help to ensure sufficient coverage of the posterior by moderating the acceptance rate of the Metropolis method. MCMC results and convergence are extensively examined by starting the scheme from many different and varied starting values.

\subsection{Forecast Evaluation}

In this section we discuss assessing the accuracy of VaR estimates and forecasts. The Basel II Accord requires financial institutions to use back-testing, so that at least one year of actual returns are compared with VaR forecasts. There are some common criteria for comparing the forecasting performance of VaR models, that is, the violations $\left(I\left(y_{t}<-V a R_{t}\right)\right)$ and the violation rate (VRate). For an in-sample period of size $n$, and forecast sample of size $m$, VRate is defined as:

$$
\text { VRate }=\frac{1}{m} \sum_{t=n+1}^{n+m} I\left(y_{t}<-V a R_{t}\right),
$$

which is simply the proportion of violating returns. Naturally, the VRate should be close to the risk level, $\alpha$, for accurate risk models.

Three formal back-testing methods for assessing forecasting performance are the unconditional coverage $(u c)$ test of Kupiec (1995), the conditional coverage $(c c)$ test of Christoffersen 
(1998), and the dynamic quantile (DQ) test of Engle and Manganelli (2004). Under the null hypothesis $\alpha=\alpha_{0}$, Kupiec (1995) employs a likelihood ratio to test whether VaR estimates, on average, provide correct coverage of the lower $\alpha$ percent tails of the forecast distributions. Christoffersen (1998) develops an independence test, employing a two-state Markov process, and combines this with the uc test to develop a joint likelihood ratio conditional coverage test, that examines whether VaR estimates display correct conditional coverage at each point in time. The conditional coverage test thus examines simultaneously whether the violations appear independently and the unconditional coverage is $\alpha$. The DQ test is also a joint test of the independence of violations and correct coverage. It employs a regression-based model of the violation-related variable 'hits', defined as $I\left(y_{t}<-V a R_{t}\right)-\alpha$, which will on average be $\alpha$ if unconditional coverage is correct. A regression-type test is then employed to examine whether the 'hits' are related to lagged 'hits', lagged VaR forecasts, or other relevant regressors, over time; a model producing accurate and independent violations and 'hits' will not be. The DQ test is well known to be more powerful than the CC test, see e.g. Berkowitz, Christofferson and Pelletier (2010).

The tests and criteria above do not consider whether the magnitude of the VaR forecasts is appropriate; only that the violations occur independently and in the right proportion. Naturally, however, it is also important to assess the accuracy of the magnitudes of the forecasts. For example, a simple method that sets VaR to be $-100 \%$ on a randomly chosen number of days, each with probability $\alpha$, and with probability $1-\alpha$ sets VaR to be $500 \%$ (say), will automatically pass all the statistical tests mentioned above, since violations occur with probability $\alpha$ and are independent over time. But this method is very poor at setting appropriate risk or capital allocation limits. We thus consider three more measures, all assessing the accuracy of the magnitude of VaR forecasts. In our opinion, these measures should be employed once it is established that a VaR forecast method passes the test above.

The Basel II Accord stipulates that market risk charges (MRC) (also called Daily Capital Charge) should also be used to assess appropriate risk models, where lower MRC is desirable. The optimization problem facing ADIs, with the number of violations and forecasts of risk as endogenous choice variables, is as follows:

Daily Capital Charge ${ }_{t}=\sup \left\{\operatorname{VaR}_{t-1},(3+k) \overline{\operatorname{VaR}_{60}}\right\}$,

where $\mathrm{VaR}_{t-1}$ is the $\mathrm{VaR}$ of the previous trading day, $\overline{\mathrm{VaR}_{60}}$ is the average VaR over the last 60 trading days, and $k$ is the penalty term from the Basel Accord Penalty Zone. The daily 
capital charge is set to be the supremum of the last trading day VaR and the average VaR over the past 60 trading days multiplied by a violation penalty weight factor $(3+k)$. Models with lower daily capital charge values are preferred for risk management. The daily capital charge attempts to give a conservative estimate of capital required to cover market risk that tries to correct for under-estimation of risk levels by applying a penalty factor to the average of previous VaR estimates. The penalty is higher the more risk has been under-estimated in the past. MRC is the average of the daily capital charges during the forecast period. McAleer and da Veiga (2008a, Table IV) displays the penalty zones at the $1 \%$ level, and the number of violations is given for 250 trading days: "Another feature of regulatory back-tests that is not easy to understand is why they require only 250 days in the back-test. With such a small sample the power of the test to reject a false hypothesis is very low indeed. So, all in all, it is highly likely that an inaccurate VaR model will pass the regulatory backtest." (Market Risk Analysis p. 336). We increase the forecast sample size here and extend the traffic light approach to obtain the penalty weight factor for such larger samples, which are given in Table 1; as discussed in detail in Section 4.

As we are also interested in the magnitude of violating returns, McAleer and da Veiga (2008a) propose the absolute deviation (AD) of violating returns, as follows:

$$
A D_{t}=\left|y_{t}-\left(-V a R_{t}\right)\right| I\left(y_{t}<-V a R_{t}\right)
$$

This measure is related to the size of the loss for violating returns. We evaluate forecast performance based on the mean and maximum of $\mathrm{AD}$, where smaller values are preferred, over the forecast sample.

Note that if models are consistently under-estimating risk and thus have too many violations, it is likely they will have smaller values for MRC, $\mathrm{AD}$ mean and $\mathrm{AD}$ maximum. Models that consistently over-estimate risk levels for violations, as the simple diabolical method mentioned above (which sets VaR randomly to $-100 \%$ thus forcing a violation), will have very large MRC, $\mathrm{AD}$ mean and $\mathrm{AD}$ maximum values. As such, models with small $\mathrm{AD}$ and MRC values are preferred only if they are generating independent violations at the correct rate $\alpha$.

Finally, the accuracy of quantile forecasts can be directly assessed using the quantile criterion loss function, given in equation (9). Here, $f_{t}(\boldsymbol{\beta})$ is replaced by the forecast VaRs for each method. The true VaR series should give the minimum of (9) and thus the most accurate model under consideration, during the forecast period, should return the minimum value of this loss function. 


\section{Empirical Applications}

In order to demonstrate and compare the forecasting performance of the proposed models, we first consider daily financial returns from five Asia-Pacific Economic Cooperation (APEC) stock markets: Standard and Poor's 500 Composite Index (U.S.), Nikkei 225 Index (Japan), TAIEX Index (Taiwan), HANG SENG Index (Hong Kong) and KOSPI Index (Korea). An equally-weighted daily return portfolio is formed from the five individual market returns, on days when all markets traded. For this portfolio, the range data from Standard and Poor's 500 Composite Index is used in the equations (6)-(8). This choice is based on the global economic scale of the US market and its strong impact on economic growth of other countries. Moreover, we consider stock market returns and their intra-day ranges. McAleer and da Veiga (2008b) compare the performance of single-index and portfolio models in forecasting VaR. All market data are obtained from Datastream International for the period January 1, 2002 to April 30, 2010. A further example concerns two exchange rate series, the Euro vs US and Japan vs US exchange rates. The range of the exchange rates were obtained from Thomson Reuters Tick History database.

The percentage returns series are calculated by taking differences of the logarithms or the daily price indices, $r_{t, j}=\left(\ln \left(P_{t}^{j}\right)-\ln \left(P_{t-1}^{j}\right)\right) \times 100$, where $P_{t}^{j}$ is the closing price index on day $t$ of asset $j$. We consider a single equal-weighted portfolio of assets, with returns: $y_{t}=\sum_{i=1}^{5} r_{t, i} \times$ $20 \%$. In addition, the differences of the logarithm of the daily series of intra-day high and low prices are taken as the range data, $R_{t}$, and are defined as $R_{t}=\left(\ln \left(R_{t, \max }\right)-\ln \left(R_{t, \min }\right)\right) \times 100$. We use the S\&P 500 range for the portfolio as the explanatory variable, and the threshold variable, in the risk models using range data, and use domestic daily range for this purpose in each individual stock market.

We examine how risk management strategies perform during the 2008-09 GFC, evaluate how the financial crisis affects risk management practices, and forecast VaR and daily capital charges, that is, diversification of more than a single investment for the purpose of risk control and management.

\subsection{VaR forecasting for the portfolio}

The full sample is divided into an in-sample period (from January 1, 2002 to July 31, 2008), and a forecast period of 400 trading days from August 1, 2008 to April 30, 2010), which covers the 2008-09 GFC. 
Table 1: Modified Traffic light approach (Basel Committee, 1996) based on 400 trading days; true coverage is $99 \%$

\begin{tabular}{llcl}
\hline Zone & $\begin{array}{l}\text { Number } \\
\text { of Violation }\end{array}$ & $\begin{array}{l}\text { Cumulative } \\
\text { probability }\end{array}$ & $\begin{array}{l}\text { Increase in scaling } \\
\text { factor }\end{array}$ \\
\hline Green & 0 & 0.01795 & 0 \\
& 1 & 0.09048 & 0 \\
& 2 & 0.23663 & 0 \\
& 3 & 0.43249 & 0 \\
& 4 & 0.62884 & 0 \\
& 5 & 0.78592 & 0 \\
& 6 & 0.89037 & 0 \\
& 7 & 0.94976 & 0 \\
\hline Yellow & 8 & 0.97923 & 0.39820 \\
& 9 & 0.99220 & 0.48142 \\
& 10 & 0.99732 & 0.56080 \\
& 11 & 0.99915 & 0.63705 \\
& 12 & 0.99975 & 0.71069 \\
\hline Red & 13 or more & 0.99993 & 1 \\
\hline
\end{tabular}


Figure 1 shows the time series plots of the portfolio returns and the S\&P500 range data: both highlight sharp increases in volatility in September, 2008 and for the subsequent early months in 2009, plus a very low volatility period approximately from 2005 to 2007; clearly the range data reflects these periods well. Table 2 presents summary statistics for each market and the portfolio returns for the full sample (contains in-sample and out-of-sample) and for the forecast (out-of-sample) sample. As expected, the forecast period displays consistently higher standard deviations and average intra-day ranges than the full sample, across all markets. Also expected, all six return series have heavy-tailed distributions and most are mildly negatively skewed. The p-values of the Jarque-Bera test for departure from normality are all very small: normality is rejected in all markets/series.

Table 2: Summary statistics: Stock index returns and ranges for five stock markets and equal weights portfolio from January 1, 2002 to April 30, 2010.

\begin{tabular}{|c|c|c|c|c|c|c|c|c|c|c|c|}
\hline \multirow[b]{2}{*}{ Statistics } & \multirow{2}{*}{$\begin{array}{c}\text { TAIEX } \\
\text { return range }\end{array}$} & \multicolumn{2}{|c|}{ Nikkei225 } & \multicolumn{2}{|c|}{ HSI } & \multicolumn{2}{|c|}{ KOSPI } & \multicolumn{2}{|c|}{ S\&P500 } & \multicolumn{2}{|c|}{ Portfolio } \\
\hline & & eturn & range & turn & range & eturn & range & return & range & eturn & range \\
\hline bst & $2055 \quad 2055$ & 2042 & 2042 & 2056 & 2056 & 2062 & 2062 & 2096 & 2096 & 1790 & 1790 \\
\hline Mean & $0.017 \quad 1.494$ & 0.001 & 1.506 & 0.030 & 1.467 & 0.043 & 1.770 & 0.001 & 1.499 & 0.005 & 1.504 \\
\hline $\mathrm{Me}$ & $0.064 \quad 1.277$ & 0.044 & 1.287 & 0.055 & 1.188 & 0.150 & 1.531 & 0.074 & 1.167 & 1.164 & 1.167 \\
\hline $\mathrm{td}$ & 1.4780 .852 & 1.618 & 0.988 & 1.662 & 1.072 & 1.639 & 1.097 & 1.391 & 1.185 & 0.056 & 1.174 \\
\hline Mir & $-6.912 \quad 0.1$ & -12.111 & 0.299 & -13.582 & 0.285 & -11.172 & 0.408 & -9.470 & 0.239 & -7.336 & 0.239 \\
\hline Мa & 6.5257 .403 & 13.235 & 11.743 & 13.407 & 17.647 & 11.284 & 15.841 & 10.957 & 10.904 & 8.29 & 10.904 \\
\hline 21 & -0.6640 .885 & -0.781 & 0.905 & -0.657 & 0.846 & -0.726 & 1.092 & -0.587 & 0.786 & -0 . & 0.797 \\
\hline Q3 & 0.7931 .851 & 0.875 & 1.844 & 0.785 & 1.761 & 0.931 & 2.108 & 0.606 & 1.811 & 0.617 & 1.818 \\
\hline Skew & $-0.257 \quad 1.653$ & -0.377 & 3.527 & 0.100 & 4.378 & -0.453 & 3.715 & -0.144 & 3.162 & -0.297 & 3.009 \\
\hline vormanty & $0.000 \quad 0.000$ & 0.000 & 0.000 & 0.000 & 0.000 & 0.000 & 0.000 & 0.000 & 0.000 & 0.000 & 0.000 \\
\hline urtosis & 2.3164 .264 & 7.433 & 23.035 & 9.345 & 39. & 4.581 & 28.018 & 9.155 & 15.320 & 6.531 & 14.031 \\
\hline \multicolumn{12}{|l|}{ Hold-out set } \\
\hline N viean & 0.0231 .8 & -0.050 & 1.914 & -0.003 & 2. & 0. & 2.109 & -0.013 & 2.33 & -0 & 2.306 \\
\hline td & 1.8251 .035 & 2.360 & 1.558 & 2.578 & 1.631 & 2.066 & 1.690 & 2.241 & 1.849 & 1.708 & 1.760 \\
\hline Minimum & -5.9330 .33 & 12.111 & 0.31 & -13.582 & 0.462 & -11.172 & 0.408 & -9.470 & 0.37 & -7.336 & 0.375 \\
\hline
\end{tabular}

The specific VaR models and methods considered are now listed:

1. Non-parametric: short-term (ST, last 25 days) and long-term (LT, last 100 days) sample percentiles. 
2. Parametric methods: GARCH(1,1) with normal and Student-t errors; RiskMetrics with normal errors and $\lambda=0.94$.

3. Semi-parametric methods: The family of CAViaR models in (1)-(8) is considered with estimation either by frequentist (denoted by "E\&M", to indicate that the same or similar estimation as in Engle and Manganelli, 2004 was used) and/or Bayesian methods, both as detailed in Section 3.

We use Fortran codes to obtain the MCMC iterates, and use the 'fminsearch' routine in the Matlab software to minimise (9) numerically. The Matlab code is adapted and updated for the RV-E\&M model from freely available code kindly provided by Simone Manganelli (downloadable from http://www.simonemanganelli.org/Simone/Research.html). The Econometrics toolbox in the software Matlab is employed to estimate both GARCH model via maximum likelihood.

For the Bayesian estimation, priors are as stated in Section 3, e.g. a uniform prior is used for the threshold value $\gamma$, that is $\gamma \sim \operatorname{Unif}(l, u)$, where $l$ and $u$ are the 1 st and 3rd quantiles of the range data. MCMC sampling is performed with 20,000 iterations in total, including the first 10,000 burn-in iterations. The last 10,000 iterations are used for inference.

We only report Bayesian estimates of the parameters for the RV, TRV and TRIG specifications in Table 3 which include posterior means, standard deviations (Std.), and the $95 \%$ credible interval (95\% CI) for each parameter. All estimated parameters are significant, except for $\beta_{3}$ of the TRV model at the $1 \%$ level and $\beta_{1}$ of the TRV model at the $5 \%$ level. The former belongs to the low range which does not respond strongly to VaR, and the latter is the intercept of the low range. Convergence of the MCMC iterates is examined via trace plots and autocorrelation function plots as diagnostic checks, not presented due to space limitations; these show that the Markov chain appears to have reached a stationary distribution in each case and indicate low autocorrelation and fairly efficient sampling and hence suggest good convergence and mixing properties of the MCMC sampling scheme. A rolling window approach is used, where a fixed in-sample size, of approximately 2000 days, is employed for estimation to forecast each day in the forecast period. Thus each method is completely re-estimated for each day in the forecast sample. This gives each method a chance to adapt to changing risk dynamics and levels.

The traffic light approach suggested by the Basel Committee (1996) deems a VaR model acceptable (green zone) if the number of violations of $1 \%$ VaR remains below the binomial $(\mathrm{p}=0.01)$ 95\% quantile. A model is disputable (yellow zone) up to the $99.99 \%$ quantile and is deemed seriously flawed (red zone) whenever more violations occur. Translated to our sample size $(\mathrm{n}=400)$ 
in Table 1, a model passes regulatory performance assessment if, at most, 7 violations occur, is disputable when between 8 and 12 violations occur and is seriously flawed for above 12 violations.

The results reported in Table 4 show numbers of violations, zone colour, VRate, VRate $/ \alpha$, $\mathrm{AD}$, Penalty Charge, MRC and the quantile criterion function, at the $1 \%$ and $5 \%$ confidence levels for the portfolio return series, for each of the $17 \mathrm{VaR}$ models/methods considered; these are informal comparison metrics. When comparing VRate/ $\alpha$, a value of 0.9 is considered better than VRate $/ \alpha=1.1$, as the loss estimates are conservative in the former and anti-conservative in the latter, case. The methods are formally tested by UC, CC and DQ, with results in Table 7 (bottom right corner). At the $1 \%$ confidence level the ST, LT, RM, both GARCH, both SAV, the AS-E\&M and the TIG-Bayesian methods are rejected by at least one test, at $5 \%$ significance. All the models using range information can not be rejected; while all the models surviving the tests are CAViaR-type models. In table 4, the top three ranked models, of those surviving the tests, for each metric are boxed. For these, the RV-Bayesian, TRV-Bayesian and TRIG-Bayesian are the top three by VRate, while these plus RV-E\&M are all in the green zone and have attracted no penalty. Looking across the metrics, the TRIG-Bayesian and RV-E\&M both rank in the top 3 models for four criteria: AD mean, Penalty, Daily Capital Charge and loss function; while RV-Bayesian, TRV-Bayesian and TRIG-Bayesian rank in the top 3 models for three criteria. Informally, these are the most accurate VaR forecast methods for the portfolio $1 \%$ risk level.

At the 5\% confidence level, again the ST, LT, RM, both GARCH and both SAV models are rejected by at least test, as are all the CAViaR models estimated via the E\&M method. As, such only the mnodels estimated via Bayesian methods survive all the tests here. For these models, the TIG, TRIG, RV and TRV-Bayesian models are closest to nominal in terms of VRate/ $\alpha$. Across the metrics, the RV-Bayesian and TRV-Bayesian also rank best on Mean AD and the criterion loss function. Note that the Basel Accord only has $1 \%$ level penalties for MRC, not $5 \%$, thus these metrics are not reported in this case.

Table 5 shows the VaR forecasting results separately for each of the five individual markets making up the portfolio. For the Nikkei225, at the $1 \%$ risk level, only three methods survive the tests, shown in Table 7: the IG-Bayesian, TIG-Bayesian and RV-Bayesian. All models under-predict risk for the Nikkei, since all VRates are larger than 0.01. The IG-Bayesian and 
Table 3: Bayesian estimation of parameters for the RV, TRV and TRIG specifications

\begin{tabular}{cccccccc}
\hline & & & & 5 & $5 \%$ & \\
Model & Parameter & Mean & Std. & $95 \%$ CI & Mean & Std. & $95 \%$ CI \\
\hline RV & $\beta_{1}$ & 0.233 & 0.042 & $(0.163,0.333)$ & 0.251 & 0.052 & $(0.157,0.360)$ \\
& $\beta_{2}$ & 0.608 & 0.035 & $(0.521,0.672)$ & 0.339 & 0.055 & $(0.235,0.445)$ \\
& $\beta_{3}$ & 0.548 & 0.044 & $(0.457,0.647)$ & 0.582 & 0.047 & $(0.490,0.671)$ \\
\hline TRV & $\beta_{1}$ & 0.221 & 0.036 & $(0.136,0.284)$ & 0.125 & 0.069 & $(-0.003,0.270)$ \\
& $\beta_{2}$ & 0.850 & 0.029 & $(0.788,0.905)$ & 0.546 & 0.104 & $(0.360,0.775)$ \\
& $\beta_{3}$ & -0.030 & 0.057 & $(-0.132,0.092)$ & 0.440 & 0.125 & $(0.168,0.659)$ \\
& $\beta_{4}$ & 1.541 & 0.196 & $(1.096,1.897)$ & 0.497 & 0.182 & $(0.139,0.846)$ \\
& $\beta_{5}$ & 0.237 & 0.073 & $(0.070,0.375)$ & 0.162 & 0.090 & $(0.015,0.360)$ \\
& $\beta_{6}$ & 0.400 & 0.051 & $(0.319,0.517)$ & 0.616 & 0.087 & $(0.426,0.778)$ \\
TRIG & $\gamma$ & 1.447 & 0.004 & $(1.435,1.452)$ & 1.424 & 0.047 & $(1.339,1.543)$ \\
\hline$\beta_{1}$ & 0.335 & 0.053 & $(0.237,0.444)$ & 0.170 & 0.082 & $(0.030,0.351)$ \\
& $\beta_{2}$ & 0.802 & 0.025 & $(0.749,0.847)$ & 0.514 & 0.108 & $(0.287,0.737)$ \\
& $\beta_{3}$ & 0.044 & 0.036 & $(0.001,0.140)$ & 0.544 & 0.167 & $(0.191,0.838)$ \\
& $\beta_{4}$ & 4.604 & 0.518 & $(3.526,5.553)$ & 1.008 & 0.405 & $(0.282,1.811)$ \\
& $\beta_{5}$ & 0.359 & 0.079 & $(0.180,0.497)$ & 0.162 & 0.094 & $(0.013,0.371)$ \\
& $\beta_{6}$ & 0.405 & 0.066 & $(0.312,0.569)$ & 0.614 & 0.091 & $(0.406,0.784)$ \\
& $\gamma$ & 1.447 & 0.004 & $(1.434,1.452)$ & 1.429 & 0.039 & $(1.365,1.548)$ \\
\hline
\end{tabular}

RV-Bayesian do best (of all models) on VRate, but IG-Bayesian and does best, of these three models, on four of the six criteria (inclding 'Zone'). For the HSI index returns, all models underpredict risk levels, but only the ad hoc ST and LT methods fail the tests. Among the surviving models, the AS-Bayesian, TCAV-Bayesian and SAV-Bayesian consistently rank in the top three models across the criteria, for HSI. For the KOSPI only the SAV, AS, IG, RV and TRV, all estimated via the Bayesian method, survive the statistical tests. Of these, the SAV, TIG and RV-Bayesian all consistently rank in the top three models over the metrics. For the TAIEX, only the SAV, IG and RV models, using both E\&M and BAyesian estimation, survive all the tests and only the RV-Bayesian is a conservative risk model (with VRate $<0.01$ ). Of these, the IG-Bayesian and IG-E\&M rank consistently well across the criteria. Finally, for the S\&P500, no model survives the tests at the $1 \%$ VaR level.

At the $5 \%$ level, a similar story ensues, see Table 6 . All models are rejected for the Nikkei225, though not at the $1 \%$ significance level for TCAV-Bayesian and TIG-Bayesian, which also do comparatively well across the criteria in Table 5. For the HSI, the ST, LT, GARCH-t, TIGBayesian and TRIG-Bayesian are all rejected. For the other models, the AS-Bayesian, TCAVBayesian and TRV-Bayesian ranked best across the criteria. For the KOSPI and the TAIEX, the IG-Bayesian and RV-Bayesian ranked most consistently among surviving models; while the 
Table 4: VaR prediction performance: using 17 model specifications and the 400 forecasts for the portfolio returns

\begin{tabular}{|c|c|c|c|c|c|c|c|c|}
\hline \multirow[t]{2}{*}{$\alpha=1 \%$} & \multirow[t]{2}{*}{ Violations } & \multirow[t]{2}{*}{ Zone } & \multirow[t]{2}{*}{ VRate $/ \alpha$} & \multicolumn{2}{|c|}{$\mathrm{AD}$} & \multirow{2}{*}{ Penalty } & \multirow{2}{*}{$\begin{array}{c}\text { Daily Capital } \\
\text { Charge }\end{array}$} & \multirow{2}{*}{$\begin{array}{c}\text { Quantile criterion } \\
\text { function }\end{array}$} \\
\hline & & & & Mean & $\operatorname{Max}$ & & & \\
\hline $\mathrm{ST}$ & 23 & Red & 5.75 & 0.724 & 1.976 & 1.000 & 12.507 & 28.360 \\
\hline LT & 11 & Yellow & 2.75 & 1.066 & 2.234 & 0.631 & 15.069 & 27.312 \\
\hline RiskMetrics & 13 & Red & 3.25 & 0.534 & 1.819 & 0.762 & 14.884 & 20.837 \\
\hline GARCH-n & 11 & Yellow & 2.75 & 0.637 & 1.829 & 0.631 & 13.014 & 20.430 \\
\hline GARCH-t & 8 & Yellow & 2.00 & 0.606 & 1.665 & 0.400 & 13.113 & 19.329 \\
\hline SAV-Bayesian & 8 & Yellow & 2.00 & 0.628 & 1.384 & 0.400 & 14.307 & 20.810 \\
\hline SAV-E\&M & 13 & Red & 3.25 & 0.823 & 2.074 & 0.762 & 13.278 & 23.462 \\
\hline AS-Bayesian & 7 & Green & 1.75 & 1.396 & 3.670 & 0.150 & 10.900 & 23.636 \\
\hline AS-E\&M & 13 & Red & 3.25 & 1.053 & 3.648 & 0.762 & 12.428 & 25.786 \\
\hline IG-Bayesian & 7 & Green & 1.75 & 0.498 & 1.676 & 0.150 & 12.283 & 18.901 \\
\hline IG-E\&M & 7 & Green & 1.75 & \begin{tabular}{|l|}
0.479 \\
\end{tabular} & 1.672 & 0.150 & 12.229 & 18.684 \\
\hline TCAV-Bayesian & 7 & Green & 1.75 & 1.277 & 4.078 & 0.150 & 11.104 & 23.022 \\
\hline TIG-Bayesian & 8 & Yellow & 2.00 & 0.739 & 1.482 & 0.400 & 12.723 & 20.177 \\
\hline RV-Bayesian & 3 & Green & 0.75 & 0.727 & 1.899 & 0.000 & 12.124 & 17.388 \\
\hline RV-E\&M & 6 & Green & 1.50 & \begin{tabular}{|l|}
0.527 \\
\end{tabular} & 1.991 & 0.000 & 11.049 & 17.092 \\
\hline TRV-Bayesian & 3 & Green & 0.75 & 0.824 & 1.825 & 0.000 & 11.719 & 17.152 \\
\hline TRIG-Bayesian & 4 & Green & 1.00 & 0.993 & 1.794 & 0.000 & 11.545 & 18.459 \\
\hline \multirow[t]{2}{*}{$\alpha=5 \%$} & Violations & VRate & VRate $/ \alpha$ & \multicolumn{2}{|c|}{$\mathrm{AD}$} & & & Quantile criterion \\
\hline & & & & Mean & $\operatorname{Max}$ & & & function \\
\hline ST & 38 & 9.50 & 1.90 & 0.809 & 2.574 & & & 28.360 \\
\hline $\mathrm{LT}$ & 21 & 5.25 & 1.05 & 1.577 & 4.241 & & & 27.312 \\
\hline RiskMetrics & 26 & 6.50 & 1.30 & 0.761 & 2.958 & & & 20.837 \\
\hline GARCH-n & 29 & 7.25 & 1.45 & 0.795 & 2.708 & & & 20.430 \\
\hline GARCH-t & 30 & 7.50 & 1.50 & 0.814 & 2.892 & & & 19.329 \\
\hline SAV-Bayesian & 26 & 6.50 & 1.30 & 0.766 & 3.013 & & & 20.810 \\
\hline SAV-E\&M & 30 & 7.50 & 1.50 & 0.837 & 2.981 & & & 23.462 \\
\hline AS-Bayesian & 25 & 6.25 & 1.25 & 0.986 & 2.300 & & & 23.636 \\
\hline AS-E\&M & 28 & 7.00 & 1.40 & 0.955 & 2.526 & & & 25.786 \\
\hline IG-Bayesian & 24 & 6.00 & 1.20 & \begin{tabular}{|l|}
0.742 \\
\end{tabular} & 2.306 & & & 18.901 \\
\hline IG-E\&M & 25 & 6.25 & 1.25 & 0.748 & 2.459 & & & 18.684 \\
\hline TCAV-Bayesian & 23 & 5.75 & 1.15 & 0.975 & \begin{tabular}{|l|}
2.292 \\
\end{tabular} & & & 23.022 \\
\hline TIG-Bayesian & 20 & 5.00 & 1.00 & 0.821 & 2.321 & & & 20.177 \\
\hline RV-Bayesian & 22 & 5.50 & 1.10 & \begin{tabular}{|l|}
0.633 \\
\end{tabular} & 2.608 & & & 17.388 \\
\hline RV-E\&M & 23 & 5.75 & 1.15 & 0.682 & 2.669 & & & 17.092 \\
\hline TRV-Bayesian & 22 & 5.50 & 1.10 & \begin{tabular}{|l|}
0.724 \\
\end{tabular} & 2.677 & & & 17.152 \\
\hline TRIG-Bayesian & 21 & 5.25 & 1.05 & 0.743 & 2.687 & & & 18.459 \\
\hline
\end{tabular}

TIG-Bayesian ranked best for the S\&P500 among surviving models.

Tables 5-6 about here

From above, we note that the CAViaR family of models are consistently dominating the models surviving the formal back-tests, while traditional methods like RM and GARCH are consistently rejected. Further, CAViaR models estimated by the Bayesian method consistently rank higher across all or most series than models estimated by the traditional method in E\&M. 
Finally, the models with IG and RV form consistently survive the tests and rank highly in most series.

We now consider two more series, both exchange rates, being the Euro vs US and Japan vs US exchange rates. Due to the availability of intra-day data for these series, the dates for sample and forecast periods are: December 21, 2004 to July 3, 2009 and July 6, 2009 to February 8, 2011. While it is well known that exchange rate returns do not, in general, exhibit significant volatility asymmetry, we choose to keep the same set of 17 models/methods, including the asymmetric ones, for consistency. Table 8 contains the p-values for the tests for the $1 \%$ and $5 \%$ VaR forecasts for these two series over the 17 methods. Tables 9-10 show the violation rates and other accuracy metrics for these two series. At the 1\% confidence level for the Euro/US series, only the two adhoc ST, LT methods fail the tests. Of the surviving models, the AS-Bayesian, TCAV-E\&M and the TRIG-Bayesian consistently rank well across the metrics. For JP/US rates at $1 \%$ risk level, the ST, SAV-E\&M, TCAV-E\&M and RV-E\&M models fails the tests. Of the surviving models, the TIG, TRIG, both Bayesian and the GARCH-t model consistently rank in the top 3 over the metrics.

At the $5 \%$ risk level, the ST, LT, RM, both GARCH models, both SAV and both AS models are rejected, for the Euro/US series. Of the remaining models, the TIG and RV, both Bayesian, consistently rank highly. For the JP/US series, only the AS models, plus RV-Bayesian and TRV-Bayesian survive the tests. Of these, AS-Bayesian and RV-Bayesian rank highest across the metrics.

Tables 9-10 about here

To summarise, for the exchange rate series, similar conclusions can be formed: the CAViaR family of models are consistently the only models surviving the formal back-tests; CAViaR models estimated by the Bayesian method rank highest across most series and; the models with IG and RV form consistently survive the tests and rank highly in most series.

Table 11 shows counts of the rejections over the three tests (UC, CC, and DQ) and across the markets and exchange rate series (counts the number of series where each, and at least one, test rejected that model) for each model. The DQ statistic is clearly the most powerful test and rejects the most models in the most markets. For the $1 \%$ VaR forecasts, the non-parametric ST and LT methods are rejected in all markets by almost all tests: clearly these are the poorest methods for this data period. The RM, GARCH with Gaussian and Student-t errors and the SAV-E\&M and AS-E\&M methods are rejected in five of the eight series. On the other hand, 
the RV-Bayesian and IG-Bayesian models are each only rejected in one series, the S\&P500, and only by the powerful DQ test, while all other methods are rejected at least three times. The detection of violations in the portfolio return series using the TRIG-Bayesian model at the $1 \%$ level is shown in Figure 2. There are four violations within the forecasting period, three of which occur during the period September-December 2008. The closeness of these violations, in time, is sufficient for the DQ test to reject this model. Other model befell a similar fate of not reacting, in time or magnitude, enough to the onset of the GFC extreme period. On the contrary, the RV-Bayesian and IG-Bayesian models, we clearly able to do so effectively across almost all of these eight series.

For the $5 \%$ VAR forecasts, table 11 shows that again the ST, LT, GARCH-t and AS-E\&M methods are the worst performed, rejected in at least 6 series. Again, the IG-Bayesian and the RV-Bayesian models perform the best, being rejected in only two out of eight series.

Table 12 shows summary statistics of the various criteria in Tables 4 and 5 for $1 \%$ VaR forecasting. While these are informal criteria, this table highlights the performance of each model across the six series and may show consistent out-performance, or otherwise. For each criteria, the mean and median across the eight series are shown. For the VRate/ $\alpha$ the square root of the average squared distance from 1 is also shown, labeled 'RMSD'. The best three models are boxed for each criterion summary, while the worst model appears in bold. For $1 \%$ VaR forecasting, clearly the non-parametric models are anti-conservative, under-estimating risk levels and performing the worst among these 17 methods across the six series. The CAViaR models are clearly the best performing as a group, since they get all the boxes except one, with two stand-outs across the criteria: the IG-Bayesian model, which is in the top three ranked models for eight of the eleven criterion summaries; and the RV-Bayesian model, in the top three ranked models for six of criterion summaries.

Table 13 shows these summaries for $5 \%$ VaR forecasting. Now it is the TRV-Bayesian, with seven top three rankings across the nine criteria, and the RV-Bayesian model, with five top three rankings, that consistently do best across the criterion summaries over the eight markets combined.

Tables 11-13 about here 


\subsection{Summary and Discussion}

Overall, the non-parametric methods have performed consistently the worst at VaR forecasting, over a range of criteria, in each of the eight series and combined across these series. The ST method's performance is perhaps explainable by the fact that 25 days is not sufficient to estimate quantiles at the $1 \%$ and $5 \%$ risk level, however the LT method's poor performance is harder to explain. Regardless, clearly these methods are not suitable for VaR forecasting at $1 \%$ and $5 \%$ levels. Further, the fully parametric RiskMetrics and GARCH models only did marginally better than the non-parametric ones, being rejected by at least one test in most or almost all series and generally and consistently under-estimating risk levels and poorly capturing risk dynamics across the data analysed.

The CAViaR models as a group did uniformly better than these four models on all metrics in almost all eight series and also in almost all metrics combined across the series, for both $1 \%$ and $5 \%$ VaR forecasting. Further, when focusing on models estimated by Bayesian or classical methods (as in E\&M), the Bayesian models also performed almost uniformly better in all markets and metrics than the same model estimated via E\&M. Gerlach et al (2011) found in simulations that the Bayesian estimates of the TCAV model parameters and forecasts of VaR were more efficient than those estimated/forecasted using classical estimation. The results here suggest that this is a more general result across the CAViaR family of models: Bayesian estimation and forecasting of CAViaR models is more efficient and accurate than classical estimation of CAViaR models, at least for those models and the data considered here. Finally, three models stood out as performing the best across the back-tests and the range of forecast accuracy criteria applied: the IG-Bayesian model and the RV-Bayesian model for 1\% VaR forecasting; and the TRV-Bayesian and RV-Bayesian models for 5\% VaR forecasting. These models consistently out-performed all others across the eight series on most of the metrics considered, at each risk level.

The Basel Committee (1996) classified the reasons for model back-testing failures into the following categories:

1. Basic integrity of the model: The system is unable to capture the risk of the positions or there is a problem in calculating volatilities and correlations.

2. Model's accuracy could be improved: Risk of some instruments not measured with sufficient precision.

3. Bad luck, or markets moved in a fashion that could not be anticipated by the model. For 
instance, volatilities or correlations turned out to be significantly different than what was predicted.

4. Intra-day trading: There is a change in positions after the VaR estimates were computed.

The US market result, where all models are rejected at VaR $1 \%$ forecasting, may be explained by point 3 ; which points to possible future research on developing a model that can capture the risk dynamics in the US market, especially during extreme or crisis conditions.

\section{Concluding Remarks}

A novel family of risk models, namely nonlinear threshold CAViaR models using intra-day price range, are proposed and the selection of optimal risk models during the 2008-09 global financial crisis is assessed and discussed. Risk management strategies and performance are assessed during this period and the performance of VaR models during the gobal financial crisis is evaluated and compared. Further, both Bayesian and frequentist (quasi-Newton) methods of estimation and forecasting are assessed and compared with real financial return data. Bayesian MCMC methods are adapted to the new family of CAViaR models, employing the link between the quantile criterion function and the Skewed-Laplace density. Five APEC stock market indices are considered, individually and via and equally weighted portfolio, and VaR is forecast for these series over roughly a two year period. Two exchange rate series are added to the analysis. The empirical evidence reveals several phenomena:

1. Risk levels and dynamics during the financial crisis seem to be predictable at a one day horizon, at least by some models (but not by others), in most markets and the portfolio considered.

2. By comparing the same model using different estimation methods, the forecasting performance of the Bayesian method is more accurate than the frequentist quasi-Newton method, in almost all cases.

3. The forecasting performance of VaR models with range information outperform models without range information, in most cases.

4. Semi-parametric models, i.e. CAViaR, consistently ranked best via statistical testing and informal assessment criteria. Next came the parametric models, though they were consistently rejected by back-tests. The non-parametric methods consistently ranked worst and were rejected in most cases. 
5. The two most favoured models for $1 \%$ VaR forecasting were the simple CAViaR models IG-Bayesian and RV-Bayesian. These were favoured by the statistical back-tests, being acceptable in almost all series, as well as by most of the informal criteria across the series.

6. The two most favoured models for $5 \%$ VaR forecasting, were the two range-based CAViaR models: TRV-Bayesian and RV-Bayesian.

7. When incorporating range information and employing the Bayesian approach, for dynamic quantile VaR forecasting, CAViaR models are competitive at worst, and far more accurate at best, when compared with a range of popular and well known VaR methods.

Each of these findings should be useful to financial practitioners and institutions. Many additional questions emerge for future research. Is the crisis period as predictable using similar time series models for longer horizons (for example 10-day forecasts)? Due to space limitations, we only focus on 1-day forecasting. Extensions to include different state variables in the information set, more than two regimes, and a smooth transition function are potential directions for further research.

Acknowledgements The authors thank three referees for their insightful comments that helped improve the paper. 


\section{References}

Alizadeh, S., Brandt, M. \& Diebold, F.X. (2002). "Range-Based Estimation of Stochastic Volatility Models," Journal of Finance, 57, 1047-1092.

Beckers, S. (1983). Variance of security price return based on high, low and closing prices. Journal of Business, 56, 97-112.

Berkowitz, J., Christofferson, P.F. \& Pelletier, D. (2011). Evaluating Value-at-Risk models with desk-level data, Management Science, to appear.

Bollerslev, T. (1986). Generalized autoregressive conditional heteroscedasticity. Journal of Econometrics, 31, 307-327.

Brandt, M. \& C. Jones (2006). Volatility Forecasting With Range-Based EGARCH Models. Journal of Business and Economic Statistics 24 (4), 470486.

Chen, C. W. S., Chiang, T. C., \& So, M. K. P. (2003). Asymmetrical reaction to US stockreturn news: evidence from major stock markets based on a double-threshold model. Journal of Economics and Business, 55, 487-502.

Chen, C.W.S., Gerlach, R., Lin, E.M.H. \& Lee, W.C.W. (2011) Bayesian Forecasting for Financial Risk Management, Pre and Post the Global Financial Crisis. Journal of Forecasting, to appear.

Chen, C.W.S., Gerlach, R., \& Lin, E.M.H. (2008). Volatility forecasting using threshold heteroskedastic models of the Intra-day Range. Computational Statistics $\mathcal{E}$ Data Analysis, $52,2990-3010$.

Chen, C.W.S., Gerlach, R., Lin, E.M.H., \& Lee, W. (2010). Bayesian forecasting for financial risk management, accepted, Journal of Forecasting.

Chen, C.W.S., \& So, M.K.P. (2006). On a threshold heteroscedastic model. International Journal of Forecasting, 22, 73-89.

Chou, R. (2005). Forecasting financial volatilities with extreme values: The conditional autoregressive range (CARR) model. Journal of Money, Credit and Banking, 37, 561-582.

Christoffersen, P. (1998). Evaluating interval forecasts. International Economic Review, 39, $841-862$ 
Engle, R.F. (1982). Autoregressive conditional heteroscedasticity with estimates of the variance of united kingdom inflation. Econometrica, 50, 987-1007.

Engle, R.F., \& Manganelli, S. (2004). CAViaR: conditional autoregressive value at risk by regression quantiles. Journal of Business $\&$ Economic Statistics, 22, 367-381.

Gallant, A. R., Hsu, C. T., \& Tauchen, G. E. (1999). Calculating volatility diffusions and extracting integrated volatility. Review of Economics and Statistics, 81, 617-631.

Garman, M.B., \& Klass, M.J. (1980). On the estimation of price volatility from historical data. Journal of Business, 53, 67-78.

Geraci, M., \& Bottai, M. (2007). Quantile regression for longitudinal data using the asymmetric Laplace distribution. Biostatistics, 8, 140-154.

Gerlach, R., \& Chen, C.W.S. (2008). Bayesian inference and model comparison for asymmetric smooth transition heteroskedastic models. Statistics and Computing, 18 (4), 391408.

Gerlach, R., Chen C.W.S., \& Chan, N.Y.C. (2011). Bayesian time-varying quantile forecasting for Value-at-Risk in financial markets, forthcoming Journal of Business E Economic Statistics.

Giacomini, R., \& Komunjer, I. (2005). Evaluation and combination of conditional quantile forecasts. Journal of Business $\&$ Economic Statistics, 23, 416-431.

Guidolin, M, \& Timmermann, A. (2006). Term structure of risk under alternative econometric specifications. Journal or Econometrics, 131, 285-308.

Haas, M., Mittnik, S. \& Paolella, M. (2006) Value-at-Risk prediction: A comparison of alternative strategies. Journal of Financial Econometrics, 4, 1, 53-89.

Hansen, B. (1994). Autoregressive Conditional Density Estimation. International Economic Review, 35, 705730 .

Hastings, W.K. (1970). Monte-carlo sampling methods using Markov chains and their applications. Biometrika, 57, 97-109.

Jorion, P. (1996). Risk: measuring the risk in Value at Risk. Financial Analysis Journal, 52, $47-56$. 
Koenker, R. \& Bassett, G. (1978). Regression quantiles. Econometrica, 1, 33-50.

Koenker, R. \& J. A. F. Machado (1999). Goodness of Fit and Related Inference Processes for Quantile Regression. Journal of the American Statistical Association, 94 (448), 1296-1310.

Kuester, K., Mittnik, S., \& Paolella, M.S. (2006). Value-at-risk prediction: a comparison of alternative strategies, Journal of Financial Econometrics, 4, 53-89.

Kupiec, P. (1995). Techniques for verifying the accuracy of risk measurement models. Journal of Derivatives, 2, 173-184.

Mandelbrot, B. (1971). When can price be arbitraged efficiently? A limit to the validity of the random walk and martingale models. Review of Economics and Statistics, 53, 225-236.

McAleer, M., \& da Veiga, B. (2008a). Forecasting value-at-risk with a parsimonious portfolio spillover GARCH (PS-GARCH) model. Journal of Forecasting, 27, 1-19.

McAleer, M., \& da Veiga, B. (2008b). Single and portfolio models for forecasting Value-at-Risk thresholds. Journal of Forecasting, 27, 217-235.

McAleer, M., Jimenez-Martin, J.-A. \& Perez-Amaral, T. (2010a). Has the Basel II Accord encouraged risk management during the 2008-09 financial crisis?, Available at SSRN: http://ssrn.com/abstract=1397239.

McAleer, M., Jimenez-Martin, J.-A. \& Perez-Amaral, T. (2010b). What happened to risk management during the 2008-09 financial crisis?, in R.W. Kolb (ed.), Lessons from the Financial Crisis: Causes, Consequences, and Our Economic Future, Wiley, New York, 307-316.

Metropolis, N., Rosenbluth, A.W., Rosenbluth, M.N., \& Teller, E. (1953). Equations of state calculations by fast computing machines. Journal of Chemical Physics, 21, 1087-1091.

Parkinson, M. (1980). The extreme value method for estimating the variance of the rate of return. Journal of Business, 53, 61-65.

Tsionas, E.G. (2003). Bayesian quantile inference. Journal of Statistical Computation and Simulation, 9, 659-674.

Yu, K., \& Moyeed, R.A. (2001). Bayesian quantile regression. Statistics and Probability Letters, $54,437-447$. 
Yu, K., \& Zhang, J. (2005). Distribution and applications - a three-parameter asymmetric laplace distribution and its extension. Communications in Statistic-Theory and Method, $34,1867-1879$.

Yu, P.L.H., Li, W.K., \& Jin, S. (2011). On some models for Value-at-Risk, forthcoming Econometric Reviews. 
Table 5: VaR prediction performance: over 450 forecasts at the $1 \%$ level for each market

\begin{tabular}{|c|c|c|c|c|c|c|c|c|}
\hline \multirow[t]{2}{*}{$\alpha=1 \%$} & & \multirow[t]{2}{*}{ Violations } & \multirow[t]{2}{*}{ Zone } & \multirow[t]{2}{*}{ VRate $/ \alpha$} & \multicolumn{2}{|c|}{$\mathrm{AD}$} & \multirow{2}{*}{$\begin{array}{c}\text { Daily Capital } \\
\text { Charge }\end{array}$} & \multirow{2}{*}{$\begin{array}{l}\text { Quantile Criterion } \\
\text { function }\end{array}$} \\
\hline & & & & & Mean & $\operatorname{Max}$ & & \\
\hline \multirow{17}{*}{ Nikkei225 } & ST & 20 & Red & 4.44 & 0.952 & 4.945 & 16.252 & 35.920 \\
\hline & LT & 11 & Yellow & 2.44 & 1.659 & 5.497 & 19.008 & 40.879 \\
\hline & RiskMetrics & 8 & Yellow & 1.78 & 1.336 & 4.492 & 15.060 & 31.756 \\
\hline & GARCH-t & 5 & Green & 1.11 & 1.609 & 4.039 & 14.556 & 29.227 \\
\hline & GARCH-n & 6 & Green & 1.33 & 1.471 & 4.218 & 15.407 & 29.657 \\
\hline & SAV-Bayesian & 8 & Yellow & 1.78 & 0.819 & 3.034 & 15.776 & 28.659 \\
\hline & SAV-E\&M & 14 & Red & 3.11 & 0.737 & 3.873 & 18.139 & 29.605 \\
\hline & AS-Bayesian & 9 & Yellow & 2.00 & 0.812 & 1.627 & 17.352 & 28.867 \\
\hline & AS-E\&M & 14 & Red & 3.11 & 0.988 & 2.077 & 17.761 & 32.905 \\
\hline & IG-Bayesian & 7 & Green & 1.56 & 0.832 & 3.263 & 15.074 & 27.009 \\
\hline & IG-E\&M & 9 & Yellow & 2.00 & 0.688 & 3.267 & 16.605 & 26.860 \\
\hline & TCAV-Bayesian & 10 & Yellow & 2.22 & 0.906 & 1.815 & 17.288 & 30.144 \\
\hline & TIG-Bayesian & 8 & Yellow & 1.78 & 1.094 & 2.277 & 14.562 & 29.212 \\
\hline & RV-Bayesian & 7 & Green & 1.56 & 0.952 & 3.191 & 14.864 & 27.455 \\
\hline & RV-E\&M & 8 & Yellow & 1.78 & 0.911 & 2.895 & 14.218 & 27.305 \\
\hline & TRV-Bayesian & 9 & Yellow & 2.00 & 0.663 & 2.907 & 17.317 & 27.279 \\
\hline & TRIG-Bayesian & 9 & Yellow & 2.00 & 0.940 & 3.688 & 16.685 & 29.158 \\
\hline \multirow[t]{17}{*}{ HSI } & ST & 21 & Red & 4.67 & 1.008 & 4.952 & 17.194 & 39.719 \\
\hline & LT & 10 & Yellow & 2.22 & 1.699 & 5.062 & 18.255 & 39.872 \\
\hline & RiskMetrics & 5 & Green & 1.11 & 1.345 & 2.400 & 16.995 & 30.880 \\
\hline & GARCH-t & 4 & Green & 0.89 & 1.179 & 1.611 & 16.449 & 30.401 \\
\hline & GARCH-n & 7 & Green & 1.56 & 0.997 & 2.311 & 17.952 & 30.263 \\
\hline & SAV-Bayesian & 5 & Green & 1.11 & 0.426 & 1.008 & 17.187 & 26.822 \\
\hline & SAV-E\&M & 4 & Green & 0.89 & 0.752 & 1.770 & 17.303 & 27.743 \\
\hline & AS-Bayesian & 3 & Green & 0.67 & 0.500 & 0.903 & 15.986 & 24.628 \\
\hline & AS-E\&M & 2 & Green & 0.44 & 0.688 & 1.117 & 16.587 & 25.235 \\
\hline & IG-Bayesian & 5 & Green & 1.11 & 0.517 & 1.341 & 16.848 & 26.750 \\
\hline & IG-E\&M & 6 & Green & 1.33 & 0.726 & 1.760 & 16.035 & 27.337 \\
\hline & TCAV-Bayesian & 6 & Green & 1.33 & 0.467 & 1.216 & 15.454 & 25.140 \\
\hline & TIG-Bayesian & 6 & Green & 1.33 & 0.635 & 1.343 & 15.337 & 25.916 \\
\hline & RV-Bayesian & 3 & Green & 0.67 & 1.099 & 1.104 & 17.984 & 28.764 \\
\hline & RV-E\&M & 5 & Green & 1.11 & 1.248 & 2.331 & 15.990 & 29.134 \\
\hline & TRV-Bayesian & 5 & Green & 1.11 & 0.688 & 1.509 & 17.570 & 28.365 \\
\hline & TRIG-Bayesian & 3 & Green & 0.67 & 1.088 & 1.232 & 17.820 & 28.507 \\
\hline \multirow[t]{17}{*}{ KOSPI } & $\mathrm{ST}$ & 22 & Red & 4.89 & 1.001 & 3.692 & 15.966 & 39.185 \\
\hline & LT & 9 & Yellow & 2.00 & 1.929 & 3.922 & 17.816 & 39.794 \\
\hline & RiskMetrics & 12 & Yellow & 2.67 & 1.151 & 3.011 & 16.081 & 33.103 \\
\hline & GARCH-t & 10 & Yellow & 2.22 & 1.044 & 2.274 & 15.402 & 32.545 \\
\hline & GARCH-n & 11 & Yellow & 2.44 & 1.234 & 2.659 & 16.236 & 30.831 \\
\hline & SAV-Bayesian & 8 & Yellow & 1.78 & 1.261 & 1.998 & 14.933 & 31.693 \\
\hline & SAV-E\&M & 10 & Yellow & 2.22 & 0.986 & 1.979 & 16.029 & 30.051 \\
\hline & AS-Bayesian & 8 & Yellow & 1.78 & 1.316 & 2.972 & 14.326 & 31.417 \\
\hline & AS-E\&M & 13 & Yellow & 2.89 & 1.152 & 3.434 & 15.096 & 33.246 \\
\hline & IG-Bayesian & 8 & Yellow & 1.78 & 1.080 & 2.211 & 15.062 & 30.435 \\
\hline & IG-E\&M & 10 & Yellow & 2.22 & 0.947 & 2.120 & 16.543 & 30.217 \\
\hline & TCAV-Bayesian & 10 & Yellow & 2.22 & 1.111 & 3.182 & 16.485 & 31.879 \\
\hline & TIG-Bayesian & 10 & Yellow & 2.22 & 1.162 & 2.889 & 16.704 & 32.655 \\
\hline & RV-Bayesian & 8 & Yellow & 1.78 & 1.196 & 3.255 & 15.244 & 31.346 \\
\hline & RV-E\&M & 10 & Yellow & 2.22 & 1.158 & 3.876 & 15.849 & 31.328 \\
\hline & TRV-Bayesian & 8 & Yellow & 1.78 & 1.345 & 2.994 & 15.541 & 32.951 \\
\hline & TRIG-Bayesian & 6 & Green & 1.33 & 1.672 & 2.597 & 16.276 & 33.138 \\
\hline
\end{tabular}


Table 5 (Continued)

\begin{tabular}{|c|c|c|c|c|c|c|c|c|}
\hline \multirow[t]{2}{*}{$\alpha=1 \%$} & & \multirow{2}{*}{ Violations } & \multirow[t]{2}{*}{ Zone } & \multirow[t]{2}{*}{ VRate $/ \alpha$} & \multicolumn{2}{|c|}{$\mathrm{AD}$} & \multirow{2}{*}{$\begin{array}{l}\text { Daily Capital } \\
\text { Charge }\end{array}$} & \multirow{2}{*}{$\begin{array}{c}\text { Quantile Criterion } \\
\text { function }\end{array}$} \\
\hline & & & & & Mean & $\operatorname{Max}$ & & \\
\hline \multirow{17}{*}{ TAIEX } & ST & 21 & Red & 4.67 & 0.801 & 1.841 & 13.911 & 32.464 \\
\hline & $\mathrm{LT}$ & 11 & Yellow & 2.44 & 0.735 & 1.315 & 14.931 & 26.866 \\
\hline & RiskMetrics & 11 & Yellow & 2.44 & 0.669 & 1.417 & 14.499 & 25.582 \\
\hline & GARCH-t & 8 & Yellow & 1.78 & 0.481 & 0.960 & 14.285 & 25.145 \\
\hline & GARCH-n & 11 & Yellow & 2.44 & 0.649 & 1.208 & 13.439 & 23.812 \\
\hline & SAV-Bayesian & 6 & Green & 1.33 & 0.471 & 0.994 & 13.415 & 22.923 \\
\hline & SAV-E\&M & 7 & Green & 1.56 & 0.442 & 0.999 & 13.174 & 22.844 \\
\hline & AS-Bayesian & 9 & Yellow & 2.00 & 0.558 & 2.042 & 14.649 & 24.434 \\
\hline & AS-E\&M & 13 & Yellow & 2.89 & 0.728 & 2.521 & 14.243 & 27.034 \\
\hline & IG-Bayesian & 6 & Green & 1.33 & 0.448 & 0.838 & 13.728 & 23.242 \\
\hline & IG-E\&M & 6 & Green & 1.33 & 0.468 & 0.908 & 13.563 & 23.114 \\
\hline & TCAV-Bayesian & 11 & Yellow & 2.44 & 0.371 & 0.942 & 15.369 & 23.597 \\
\hline & TIG-Bayesian & 9 & Yellow & 2.00 & 0.613 & 1.733 & 15.043 & 25.371 \\
\hline & RV-Bayesian & 4 & Green & 0.89 & 0.737 & 1.255 & 13.690 & 23.308 \\
\hline & RV-E\&M & 5 & Green & 1.11 & 0.643 & 1.217 & 13.487 & 23.291 \\
\hline & TRV-Bayesian & 6 & Green & 1.33 & 0.931 & 1.944 & 13.731 & 26.026 \\
\hline & TRIG-Bayesian & 6 & Green & 1.33 & 0.783 & 2.248 & 13.695 & 25.123 \\
\hline \multirow[t]{17}{*}{ S\&P500 } & $\mathrm{ST}$ & 24 & Red & 5.33 & 0.958 & 4.390 & 15.903 & 39.708 \\
\hline & LT & 11 & Yellow & 2.44 & 1.205 & 4.390 & 18.777 & 35.553 \\
\hline & RiskMetrics & 13 & Yellow & 2.89 & 0.648 & 3.749 & 17.522 & 28.498 \\
\hline & GARCH-t & 9 & Yellow & 2.00 & 0.713 & 3.437 & 18.200 & 28.410 \\
\hline & GARCH-n & 14 & Red & 3.11 & 0.656 & 3.850 & 16.726 & 27.212 \\
\hline & SAV-Bayesian & 10 & Yellow & 2.22 & 0.854 & 4.008 & 16.379 & 28.492 \\
\hline & SAV-E\&M & 13 & Yellow & 2.89 & 1.178 & 4.962 & 16.597 & 34.410 \\
\hline & AS-Bayesian & 11 & Yellow & 2.44 & 0.790 & 3.926 & 16.450 & 28.306 \\
\hline & AS-E\&M & 15 & Red & 3.33 & 0.986 & 4.828 & 16.658 & 32.543 \\
\hline & IG-Bayesian & 9 & Yellow & 2.00 & 0.860 & 3.482 & 16.369 & 28.084 \\
\hline & IG-E\&M & 11 & Yellow & 2.44 & 0.878 & 4.227 & 17.221 & 30.176 \\
\hline & TCAV-Bayesian & 12 & Yellow & 2.67 & 0.963 & 3.746 & 17.123 & 31.511 \\
\hline & TIG-Bayesian & 11 & Yellow & 2.44 & 0.701 & 3.702 & 17.029 & 28.016 \\
\hline & RV-Bayesian & 9 & Yellow & 2.00 & 0.849 & 3.976 & 15.385 & 26.847 \\
\hline & RV-E\&M & 17 & Red & 3.77 & 0.835 & 4.680 & 16.061 & 31.357 \\
\hline & TRV-Bayesian & 10 & Yellow & 2.22 & 1.028 & 4.286 & 15.409 & 29.033 \\
\hline & TRIG-Bayesian & 11 & Yellow & 2.44 & 1.010 & 5.015 & 15.657 & 29.835 \\
\hline
\end{tabular}


Table 6: VaR prediction performance: over 450 forecasts at the $5 \%$ level for each market

\begin{tabular}{|c|c|c|c|c|c|c|c|}
\hline \multirow[t]{2}{*}{$\alpha=5 \%$} & & \multirow[t]{2}{*}{ Violations } & \multirow[t]{2}{*}{ VRate } & \multirow[t]{2}{*}{ VRate $/ \alpha$} & \multicolumn{2}{|c|}{$\mathrm{AD}$} & \multirow{2}{*}{$\begin{array}{l}\text { Quantile Criterior } \\
\text { function }\end{array}$} \\
\hline & & & & & Mean & $\operatorname{Max}$ & \\
\hline \multirow[t]{17}{*}{ Nikkei225 } & $\mathrm{ST}$ & 39 & 8.67 & 1.73 & 0.926 & 5.532 & 35.920 \\
\hline & LT & 20 & 4.44 & 0.89 & 2.280 & 8.979 & 40.879 \\
\hline & RiskMetrics & 34 & 7.56 & 1.51 & 1.002 & 6.062 & 31.756 \\
\hline & GARCH-t & 36 & 8.00 & 1.60 & 1.022 & 5.868 & 29.227 \\
\hline & GARCH-n & 34 & 7.56 & 1.51 & 1.054 & 5.961 & 29.657 \\
\hline & SAV-Bayesian & 29 & 6.45 & 1.29 & 0.958 & 5.564 & 28.659 \\
\hline & SAV-E\&M & 34 & 7.56 & 1.51 & 1.040 & 6.101 & 29.605 \\
\hline & AS-Bayesian & 32 & 7.11 & 1.42 & 0.914 & 4.379 & 28.867 \\
\hline & AS-E\&M & 33 & 7.33 & 1.47 & 1.038 & 4.810 & 32.905 \\
\hline & IG-Bayesian & 32 & 7.11 & 1.42 & 0.851 & 5.748 & 27.009 \\
\hline & IG-E\&M & 32 & 7.11 & 1.42 & 0.958 & 5.942 & 26.860 \\
\hline & TCAV-Bayesian & 28 & 6.22 & 1.24 & 1.016 & 3.991 & 30.144 \\
\hline & TIG-Bayesian & 28 & 6.22 & 1.24 & 0.986 & 4.081 & 29.212 \\
\hline & RV-Bayesian & 33 & 7.33 & 1.47 & 0.926 & 5.242 & 27.455 \\
\hline & RV-E\&M & 36 & 8.00 & 1.60 & 1.113 & 5.676 & 27.305 \\
\hline & TRV-Bayesian & 30 & 6.67 & 1.33 & 0.825 & 3.986 & 27.279 \\
\hline & TRIG-Bayesian & 34 & 7.56 & 1.51 & 0.899 & 5.470 & 29.158 \\
\hline \multirow[t]{17}{*}{ HSI } & $\mathrm{ST}$ & 40 & 8.89 & 1.78 & 0.972 & 5.275 & 39.719 \\
\hline & $\mathrm{LT}$ & 25 & 5.56 & 1.11 & 1.536 & 8.475 & 39.872 \\
\hline & RiskMetrics & 22 & 4.89 & 0.98 & 1.162 & 5.675 & 30.880 \\
\hline & GARCH-t & 27 & 6.00 & 1.20 & 1.058 & 5.893 & 30.401 \\
\hline & GARCH-n & 25 & 5.56 & 1.11 & 1.041 & 5.613 & 30.263 \\
\hline & SAV-Bayesian & 24 & 5.33 & 1.04 & 0.810 & 4.240 & 26.822 \\
\hline & SAV-E\&M & 22 & 4.89 & 0.98 & 1.014 & 5.187 & 27.743 \\
\hline & AS-Bayesian & 28 & 6.22 & 1.24 & 0.660 & 2.751 & 24.628 \\
\hline & AS-E\&M & 22 & 4.89 & 0.98 & 0.814 & 4.147 & 25.235 \\
\hline & IG-Bayesian & 25 & 5.55 & 1.11 & 0.854 & 4.573 & 26.750 \\
\hline & IG-E\&M & 24 & 5.33 & 1.07 & 0.945 & 5.277 & 27.337 \\
\hline & TCAV-Bayesian & 27 & 6.00 & 1.20 & 0.676 & 2.779 & 25.140 \\
\hline & TIG-Bayesian & 29 & 6.44 & 1.29 & 0.795 & 3.556 & 25.916 \\
\hline & RV-Bayesian & 22 & 4.89 & 0.98 & 0.987 & 4.356 & 28.764 \\
\hline & RV-E\&M & 22 & 4.89 & 0.98 & 1.011 & 5.232 & 29.134 \\
\hline & TRV-Bayesian & 25 & 5.56 & 1.11 & 0.735 & 2.912 & 28.365 \\
\hline & TRIG-Bayesian & 28 & 6.22 & 1.24 & 0.824 & 4.480 & 28.507 \\
\hline \multirow[t]{17}{*}{ KOSPI } & $\mathrm{ST}$ & 38 & 8.44 & 1.69 & 1.213 & 4.483 & 39.185 \\
\hline & $\mathrm{LT}$ & 22 & 4.88 & 0.98 & 1.942 & 4.302 & 39.794 \\
\hline & RiskMetrics & 30 & 6.67 & 1.33 & 1.181 & 5.033 & 33.103 \\
\hline & GARCH-t & 30 & 6.67 & 1.33 & 1.225 & 4.921 & 32.545 \\
\hline & GARCH-n & 28 & 6.22 & 1.24 & 1.311 & 4.784 & 30.831 \\
\hline & SAV-Bayesian & 28 & 6.22 & 1.24 & 1.252 & 4.604 & 31.693 \\
\hline & SAV-E\&M & 30 & 6.67 & 1.33 & 1.176 & 4.671 & 30.051 \\
\hline & AS-Bayesian & 28 & 6.22 & 1.24 & 1.243 & 4.695 & 31.417 \\
\hline & AS-E\&M & 28 & 6.22 & 1.24 & 1.204 & 4.592 & 33.246 \\
\hline & IG-Bayesian & 27 & 6.00 & 1.20 & 1.168 & 4.329 & 30.435 \\
\hline & IG-E\&M & 26 & 5.78 & 1.16 & 1.190 & 4.325 & 30.217 \\
\hline & TCAV-Bayesian & 28 & 6.22 & 1.24 & 1.197 & 4.702 & 31.879 \\
\hline & TIG-Bayesian & 26 & 5.78 & 1.16 & 1.217 & 4.586 & 32.655 \\
\hline & RV-Bayesian & 23 & 5.11 & 1.02 & 1.169 & 5.321 & 31.346 \\
\hline & RV-E\&M & 23 & 5.11 & 1.02 & 1.235 & 5.478 & 31.328 \\
\hline & TRV-Bayesian & 23 & 5.11 & 1.02 & 1.319 & 5.446 & 32.951 \\
\hline & TRIG-Bayesian & 24 & 5.33 & 1.07 & 1.358 & 5.287 & 33.138 \\
\hline
\end{tabular}


Table 6 (Continued)

\begin{tabular}{|c|c|c|c|c|c|c|c|}
\hline \multirow[t]{2}{*}{$\alpha=5 \%$} & & \multirow[t]{2}{*}{ Violations } & \multirow[t]{2}{*}{ VRate } & \multirow[t]{2}{*}{ VRate $/ \alpha$} & \multicolumn{2}{|c|}{$\mathrm{AD}$} & \multirow{2}{*}{$\begin{array}{c}\text { Quantile Criterior } \\
\text { function }\end{array}$} \\
\hline & & & & & Mean & $\operatorname{Max}$ & \\
\hline \multirow[t]{17}{*}{ TAIEX } & ST & 44 & 9.78 & 1.96 & 0.802 & 2.223 & 32.464 \\
\hline & $\mathrm{LT}$ & 25 & 5.56 & 1.11 & 1.053 & 2.761 & 26.866 \\
\hline & RiskMetrics & 29 & 6.44 & 1.29 & 0.819 & 2.289 & 25.582 \\
\hline & GARCH-t & 29 & 6.44 & 1.29 & 0.884 & 2.274 & 25.145 \\
\hline & GARCH-n & 28 & 6.22 & 1.24 & 0.877 & 2.192 & 23.812 \\
\hline & SAV-Bayesian & 26 & 5.78 & 1.56 & 0.847 & 2.199 & 22.923 \\
\hline & SAV-E\&M & 28 & 6.22 & 1.24 & 0.833 & 2.191 & 22.844 \\
\hline & AS-Bayesian & 29 & 6.44 & 1.29 & 0.858 & 2.944 & 24.434 \\
\hline & AS-E\&M & 33 & 7.33 & 1.47 & 0.876 & 3.424 & 27.034 \\
\hline & IG-Bayesian & 25 & 5.56 & 1.11 & 0.883 & 2.016 & 23.242 \\
\hline & IG-E\&M & 27 & 6.00 & 1.20 & 0.881 & 2.272 & 23.114 \\
\hline & TCAV-Bayesian & 30 & 6.67 & 1.33 & 0.724 & 2.967 & 23.597 \\
\hline & TIG-Bayesian & 31 & 6.89 & 1.38 & 0.811 & 1.966 & 25.371 \\
\hline & RV-Bayesian & 23 & 5.11 & 1.02 & 0.850 & 2.111 & 23.308 \\
\hline & RV-E\&M & 26 & 5.78 & 1.16 & 0.884 & 2.351 & 23.291 \\
\hline & TRV-Bayesian & 24 & 5.33 & 1.07 & 0.928 & 2.246 & 26.026 \\
\hline & TRIG-Bayesian & 26 & 5.78 & 1.56 & 0.817 & 2.538 & 25.123 \\
\hline \multirow[t]{17}{*}{ S\&P500 } & $\mathrm{ST}$ & 43 & 9.56 & 1.91 & 1.047 & 4.577 & 39.708 \\
\hline & LT & 29 & 6.44 & 1.29 & 1.397 & 6.176 & 35.553 \\
\hline & RiskMetrics & 29 & 6.44 & 1.29 & 1.139 & 5.352 & 28.498 \\
\hline & GARCH-t & 31 & 6.89 & 1.38 & 1.169 & 5.423 & 28.410 \\
\hline & GARCH-n & 31 & 6.89 & 1.38 & 1.175 & 5.418 & 27.212 \\
\hline & SAV-Bayesian & 31 & 6.89 & 1.38 & 1.110 & 5.541 & 28.492 \\
\hline & SAV-E\&M & 30 & 6.67 & 1.33 & 1.231 & 5.885 & 34.410 \\
\hline & AS-Bayesian & 31 & 6.89 & 1.38 & 1.241 & 6.060 & 28.306 \\
\hline & AS-E\&M & 40 & 8.89 & 1.78 & 1.296 & 6.374 & 32.543 \\
\hline & IG-Bayesian & 29 & 6.44 & 1.29 & 1.153 & 5.370 & 28.084 \\
\hline & IG-E\&M & 31 & 6.89 & 1.38 & 1.156 & 5.546 & 30.176 \\
\hline & TCAV-Bayesian & 30 & 6.67 & 1.33 & 1.060 & 5.137 & 31.511 \\
\hline & TIG-Bayesian & 30 & 6.67 & 1.33 & 1.061 & 5.034 & 28.016 \\
\hline & RV-Bayesian & 33 & 7.33 & 1.47 & 0.997 & 5.602 & 26.847 \\
\hline & RV-E\&M & 41 & 9.11 & 1.82 & 1.141 & 5.879 & 31.357 \\
\hline & TRV-Bayesian & 34 & 7.56 & 1.51 & 0.943 & 6.005 & 29.033 \\
\hline & TRIG-Bayesian & 33 & 7.33 & 1.51 & 0.970 & 5.786 & 29.835 \\
\hline
\end{tabular}


Table 7: P-values of the unconditional and conditional coverage tests, and Dynamic Quantile tests

\begin{tabular}{|c|c|c|c|c|c|c|c|c|c|c|c|c|}
\hline \multirow[b]{4}{*}{$\mathrm{ST}$} & \multicolumn{6}{|c|}{ Nikkei225 } & \multicolumn{6}{|c|}{ HSI } \\
\hline & \multicolumn{3}{|c|}{$1 \%$} & \multicolumn{3}{|c|}{$5 \%$} & \multicolumn{3}{|c|}{$1 \%$} & \multicolumn{3}{|c|}{$5 \%$} \\
\hline & $L R_{u c}$ & $L R_{c c}$ & DQ & $L R_{u c}$ & $L R_{c c}$ & DQ & $L R_{u c}$ & $L R_{c c}$ & DQ & $L R_{u c}$ & $L R_{c c}$ & DQ \\
\hline & 0.000 & 0.000 & 0.000 & 0.001 & 0.003 & 0.000 & 0.000 & 0.000 & 0.000 & 0.001 & 0.002 & 0.000 \\
\hline $\mathrm{LT}$ & 0.009 & 0.025 & 0.000 & 0.582 & 0.146 & 0.000 & 0.025 & 0.004 & 0.000 & 0.595 & 0.126 & 0.000 \\
\hline RiskMetrics & 0.135 & 0.281 & 0.000 & 0.020 & 0.061 & 0.005 & 0.816 & 0.919 & 0.740 & 0.914 & 0.697 & 0.156 \\
\hline GARCH-n & 0.499 & 0.731 & 0.000 & 0.020 & 0.032 & 0.004 & 0.273 & 0.489 & 0.331 & 0.595 & 0.756 & 0.194 \\
\hline GARCH-t & 0.816 & 0.919 & 0.000 & 0.007 & 0.021 & 0.001 & 0.809 & 0.938 & 0.562 & 0.345 & 0.604 & 0.044 \\
\hline SAV-Bayesian & 0.135 & 0.281 & 0.001 & 0.177 & 0.302 & 0.004 & 0.816 & 0.919 & 0.607 & 0.748 & 0.779 & 0.632 \\
\hline SAV-E\&M & 0.000 & 0.001 & 0.000 & 0.020 & 0.032 & 0.001 & 0.809 & 0.938 & 0.615 & 0.914 & 0.697 & 0.181 \\
\hline AS-Bayesian & 0.061 & 0.142 & 0.043 & 0.053 & 0.090 & 0.006 & 0.449 & 0.739 & 0.564 & 0.251 & 0.414 & 0.073 \\
\hline AS-E\&M & 0.000 & 0.001 & 0.000 & 0.033 & 0.055 & 0.004 & 0.183 & 0.411 & 0.366 & 0.914 & 0.697 & 0.184 \\
\hline IG-Bayesian & 0.273 & 0.489 & 0.243 & 0.053 & 0.090 & 0.006 & 0.816 & 0.919 & 0.653 & 0.595 & 0.756 & 0.214 \\
\hline IG-E\&M & 0.061 & 0.142 & 0.043 & 0.053 & 0.013 & 0.002 & 0.499 & 0.731 & 0.464 & 0.748 & 0.779 & 0.224 \\
\hline TCAV-Bayesian & 0.025 & 0.063 & 0.007 & 0.251 & 0.079 & 0.047 & 0.499 & 0.731 & 0.595 & 0.345 & 0.542 & 0.085 \\
\hline TIG-Bayesian & 0.135 & 0.281 & 0.132 & 0.251 & 0.079 & 0.037 & 0.499 & 0.731 & 0.283 & 0.177 & 0.395 & 0.026 \\
\hline RV-Bayesian & 0.273 & 0.489 & 0.239 & 0.033 & 0.096 & 0.006 & 0.449 & 0.739 & 0.753 & 0.914 & 0.992 & 0.180 \\
\hline RV-E\&M & 0.135 & 0.281 & 0.000 & 0.007 & 0.021 & 0.000 & 0.816 & 0.919 & 0.432 & 0.914 & 0.697 & 0.176 \\
\hline TRV-Bayesian & 0.061 & 0.142 & 0.025 & 0.122 & 0.210 & 0.009 & 0.816 & 0.919 & 0.535 & 0.595 & 0.756 & 0.027 \\
\hline \multirow{4}{*}{ TRIG-Bayesian } & 0.061 & 0.142 & 0.036 & 0.020 & 0.061 & 0.008 & 0.449 & 0.739 & 0.610 & 0.251 & 0.144 & 0.010 \\
\hline & \multicolumn{6}{|c|}{ KOSPI } & \multicolumn{6}{|c|}{ TAIEX } \\
\hline & \multicolumn{3}{|c|}{$1 \%$} & \multicolumn{3}{|c|}{$5 \%$} & \multicolumn{3}{|c|}{$1 \%$} & & $5 \%$ & \\
\hline & $L R_{u c}$ & $L R_{c c}$ & DQ & $L R_{u c}$ & $L R_{c c}$ & DQ & $L R_{u c}$ & $L R_{c c}$ & DQ & $L R_{u c}$ & $L R_{c c}$ & DQ \\
\hline $\mathrm{ST}$ & 0.000 & 0.000 & 0.000 & 0.002 & 0.006 & 0.000 & 0.000 & 0.000 & 0.000 & 0.000 & 0.000 & 0.000 \\
\hline $\mathrm{LT}$ & 0.061 & 0.064 & 0.000 & 0.914 & 0.697 & 0.001 & 0.009 & 0.018 & 0.000 & 0.595 & 0.806 & 0.101 \\
\hline RiskMetrics & 0.003 & 0.009 & 0.000 & 0.122 & 0.297 & 0.158 & 0.009 & 0.025 & 0.000 & 0.177 & 0.284 & 0.056 \\
\hline GARCH-n & 0.009 & 0.025 & 0.002 & 0.251 & 0.414 & 0.104 & 0.009 & 0.025 & 0.000 & 0.251 & 0.331 & 0.071 \\
\hline GARCH-t & 0.025 & 0.063 & 0.007 & 0.122 & 0.210 & 0.094 & 0.135 & 0.281 & 0.000 & 0.177 & 0.284 & 0.061 \\
\hline SAV-Bayesian & 0.135 & 0.281 & 0.111 & 0.251 & 0.414 & 0.183 & 0.499 & 0.731 & 0.438 & 0.460 & 0.694 & 0.196 \\
\hline SAV-E\&M & 0.025 & 0.063 & 0.007 & 0.122 & 0.210 & 0.104 & 0.273 & 0.489 & 0.275 & 0.251 & 0.331 & 0.070 \\
\hline AS-Bayesian & 0.135 & 0.281 & 0.125 & 0.251 & 0.414 & 0.002 & 0.061 & 0.142 & 0.026 & 0.177 & 0.302 & 0.018 \\
\hline AS-E\&M & 0.001 & 0.003 & 0.000 & 0.251 & 0.414 & 0.001 & 0.001 & 0.003 & 0.000 & 0.033 & 0.055 & 0.000 \\
\hline IG-Bayesian & 0.135 & 0.281 & 0.127 & 0.345 & 0.542 & 0.145 & 0.499 & 0.731 & 0.251 & 0.595 & 0.756 & 0.232 \\
\hline IG-E\&M & 0.025 & 0.063 & 0.007 & 0.460 & 0.678 & 0.198 & 0.499 & 0.731 & 0.278 & 0.345 & 0.604 & 0.168 \\
\hline TCAV-Bayesian & 0.025 & 0.063 & 0.010 & 0.251 & 0.414 & 0.001 & 0.009 & 0.025 & 0.001 & 0.122 & 0.210 & 0.017 \\
\hline TIG-Bayesian & 0.025 & 0.063 & 0.007 & 0.460 & 0.152 & 0.019 & 0.061 & 0.142 & 0.013 & 0.081 & 0.140 & 0.006 \\
\hline RV-Bayesian & 0.135 & 0.281 & 0.088 & 0.914 & 0.287 & 0.220 & 0.809 & 0.938 & 0.177 & 0.914 & 0.978 & 0.516 \\
\hline RV-E\&M & 0.025 & 0.063 & 0.007 & 0.914 & 0.978 & 0.441 & 0.816 & 0.919 & 0.193 & 0.460 & 0.694 & 0.514 \\
\hline TRV-Bayesian & 0.135 & 0.281 & 0.027 & 0.914 & 0.978 & 0.472 & 0.499 & 0.731 & 0.026 & 0.748 & 0.779 & 0.198 \\
\hline TRIG-Bayesian & 0.499 & 0.731 & 0.300 & 0.748 & 0.912 & 0.287 & 0.499 & 0.731 & 0.022 & 0.460 & 0.694 & 0.369 \\
\hline & & & & 500 & & & & & Por & & & \\
\hline & & $1 \%$ & & & $5 \%$ & & & $1 \%$ & & & $5 \%$ & \\
\hline & $L R_{u c}$ & $L R_{c c}$ & DQ & $L R_{u c}$ & $L R_{c c}$ & DQ & $L R_{u c}$ & $L R_{c c}$ & DQ & $L R_{u c}$ & $L R_{c c}$ & DQ \\
\hline $\mathrm{ST}$ & 0.000 & 0.000 & 0.000 & 0.000 & 0.000 & 0.000 & 0.000 & 0.000 & 0.000 & 0.000 & 0.001 & 0.000 \\
\hline LT & 0.009 & 0.025 & 0.000 & 0.177 & 0.271 & 0.000 & 0.004 & 0.009 & 0.000 & 0.820 & 0.267 & 0.000 \\
\hline RiskMetrics & 0.001 & 0.003 & 0.000 & 0.177 & 0.312 & 0.035 & 0.000 & 0.001 & 0.000 & 0.187 & 0.400 & 0.040 \\
\hline GARCH-n & 0.000 & 0.001 & 0.000 & 0.081 & 0.146 & 0.028 & 0.004 & 0.009 & 0.000 & 0.052 & 0.148 & 0.015 \\
\hline GARCH-t & 0.061 & 0.142 & 0.000 & 0.081 & 0.146 & 0.028 & 0.077 & 0.176 & 0.022 & 0.032 & 0.096 & 0.007 \\
\hline SAV-Bayesian & 0.025 & 0.063 & 0.000 & 0.081 & 0.146 & 0.006 & 0.077 & 0.070 & 0.000 & 0.187 & 0.400 & 0.092 \\
\hline SAV-E\&M & 0.001 & 0.003 & 0.000 & 0.122 & 0.218 & 0.009 & 0.000 & 0.001 & 0.000 & 0.032 & 0.059 & 0.005 \\
\hline AS-Bayesian & 0.009 & 0.025 & 0.000 & 0.081 & 0.146 & 0.018 & 0.173 & 0.346 & 0.084 & 0.269 & 0.502 & 0.098 \\
\hline AS-E\&M & 0.000 & 0.000 & 0.000 & 0.001 & 0.003 & 0.000 & 0.000 & 0.001 & 0.000 & 0.083 & 0.164 & 0.011 \\
\hline IG-Bayesian & 0.061 & 0.142 & 0.000 & 0.177 & 0.302 & 0.068 & 0.173 & 0.346 & 0.105 & 0.373 & 0.596 & 0.071 \\
\hline IG-E\&M & 0.009 & 0.025 & 0.000 & 0.081 & 0.146 & 0.012 & 0.173 & 0.346 & 0.079 & 0.269 & 0.502 & 0.044 \\
\hline TCAV-Bayesian & 0.003 & 0.009 & 0.000 & 0.122 & 0.210 & 0.108 & 0.173 & 0.346 & 0.111 & 0.501 & 0.667 & 0.223 \\
\hline TIG-Bayesian & 0.009 & 0.025 & 0.000 & 0.122 & 0.210 & 0.052 & 0.077 & 0.176 & 0.049 & 1.000 & 0.645 & 0.232 \\
\hline RV-Bayesian & 0.061 & 0.142 & 0.000 & 0.033 & 0.055 & 0.024 & 0.599 & 0.853 & 0.642 & 0.651 & 0.878 & 0.295 \\
\hline RV-E\&M & 0.000 & 0.000 & 0.000 & 0.000 & 0.001 & 0.000 & 0.349 & 0.586 & 0.425 & 0.501 & 0.318 & 0.015 \\
\hline TRV-Bayesian & 0.025 & 0.063 & 0.000 & 0.020 & 0.061 & 0.004 & 0.599 & 0.853 & 0.772 & 0.651 & 0.878 & 0.293 \\
\hline TRIG-Bayesian & 0.009 & 0.025 & 0.000 & 0.033 & 0.055 & 0.023 & 1.000 & 0.960 & 0.561 & 0.820 & 0.302 & 0.177 \\
\hline
\end{tabular}


Table 8: P-values of the unconditional and conditional coverage tests, and Dynamic Quantile test for each exchange rate series

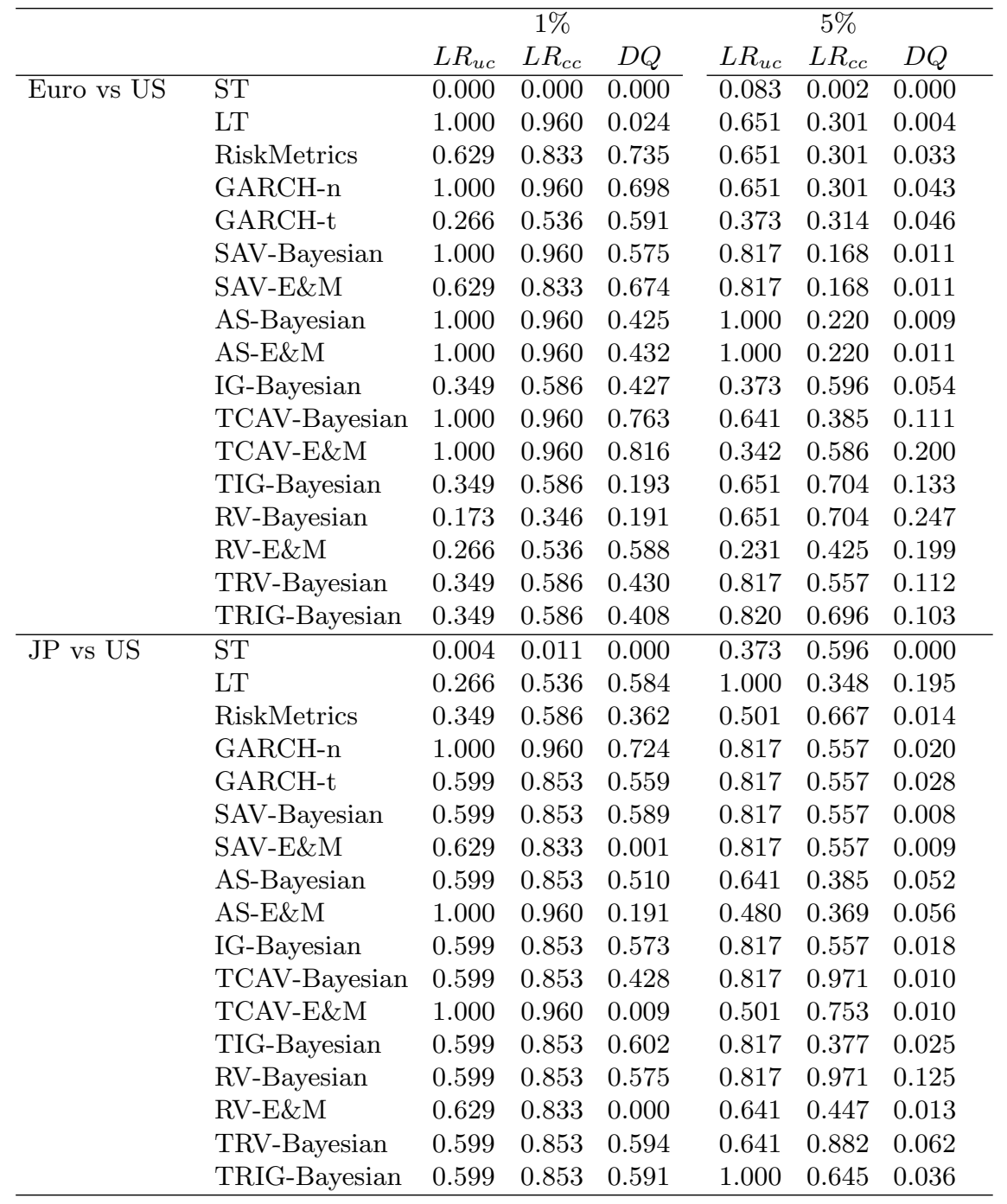


Table 9: VaR prediction performance: using 17 model specifications and the 400 forecasts for the exchange returns

\begin{tabular}{|c|c|c|c|c|c|c|c|c|c|}
\hline \multicolumn{2}{|l|}{$\alpha=1 \%$} & \multirow[t]{2}{*}{ Violations } & \multirow[t]{2}{*}{ Zone } & \multirow[t]{2}{*}{ VRate $/ \alpha$} & \multicolumn{2}{|c|}{$\mathrm{AD}$} & \multicolumn{3}{|c|}{ Penalty Daily Capital Quantile criterion } \\
\hline & & & & & Mean & $\operatorname{Max}$ & & Charge & function \\
\hline \multirow{17}{*}{ Euro vs US } & ST & 19 & Red & 4.75 & 0.297 & 0.803 & 1.000 & 5.508 & 11.074 \\
\hline & LT & 4 & Green & 1.00 & 0.390 & 0.879 & 0.000 & 5.108 & 8.358 \\
\hline & RiskMetrics & 5 & Green & 1.25 & 0.196 & 0.392 & 0.000 & 4.756 & 7.242 \\
\hline & GARCH-n & 4 & Green & 1.00 & 0.246 & 0.453 & 0.000 & 4.816 & 7.314 \\
\hline & GARCH-t & 2 & Green & 0.50 & 0.374 & 0.398 & 0.000 & 5.054 & 7.436 \\
\hline & SAV-Bayesian & 4 & Green & 1.00 & 0.220 & 0.367 & 0.000 & 4.769 & 7.157 \\
\hline & SAV-E\&M & 5 & Green & 1.25 & 0.199 & 0.405 & 0.000 & 4.766 & 7.266 \\
\hline & AS-Bayesian & 4 & Green & 1.00 & 0.166 & 0.247 & 0.000 & 4.827 & 7.002 \\
\hline & AS-E\&M & 4 & Green & 1.00 & 0.175 & 0.258 & 0.000 & 4.819 & 7.024 \\
\hline & IG-Bayesian & 6 & Green & 1.50 & 0.243 & 0.447 & 0.000 & 4.560 & 7.474 \\
\hline & TCAV-Bayesian & 4 & Green & 1.00 & 0.319 & 0.466 & 0.000 & 4.828 & 7.611 \\
\hline & TCAV-E\&M & 4 & Green & 1.00 & 0.167 & 0.227 & 0.000 & 4.916 & 7.105 \\
\hline & TIG-Bayesian & 6 & Green & 1.50 & 0.242 & 0.483 & 0.000 & 4.662 & 7.583 \\
\hline & RV-Bayesian & 7 & Green & 1.75 & 0.159 & 0.520 & 0.000 & 4.438 & 7.014 \\
\hline & RV-E\&M & 2 & Green & 0.50 & 0.327 & 0.381 & 0.000 & 5.157 & 7.452 \\
\hline & TRV-Bayesian & 6 & Green & 1.50 & 0.177 & 0.447 & 0.000 & 4.517 & 7.082 \\
\hline & TRIG-Bayesian & 6 & Green & 1.50 & 0.166 & 0.443 & 0.000 & 4.502 & 7.000 \\
\hline \multirow[t]{17}{*}{ JP vs US } & ST & 11 & Yellow & 2.75 & 0.442 & 1.003 & 0.637 & 5.067 & 10.364 \\
\hline & LT & 2 & Green & 0.50 & 0.478 & 0.608 & 0.000 & 5.399 & 8.163 \\
\hline & RiskMetrics & 6 & Green & 1.50 & 0.472 & 1.132 & 0.000 & 4.696 & 8.882 \\
\hline & GARCH-n & 4 & Green & 1.00 & 0.497 & 0.683 & 0.000 & 4.780 & 8.222 \\
\hline & GARCH-t & 3 & Green & 0.75 & 0.427 & 0.529 & 0.000 & 5.305 & 8.206 \\
\hline & SAV-Bayesian & 3 & Green & 0.75 & 0.343 & 0.495 & 0.000 & 5.567 & 8.311 \\
\hline & SAV-E\&M & 5 & Green & 1.25 & 0.579 & 1.193 & 0.000 & 5.101 & 9.643 \\
\hline & AS-Bayesian & 3 & Green & 0.75 & 0.339 & 0.436 & 0.000 & 5.939 & 8.730 \\
\hline & AS-E\&M & 4 & Green & 1.00 & 0.331 & 0.437 & 0.000 & 5.852 & 8.933 \\
\hline & IG-Bayesian & 3 & Green & 0.75 & 0.371 & 0.448 & 0.000 & 5.561 & 8.369 \\
\hline & TCAV-Bayesian & 3 & Green & 0.75 & 0.470 & 0.596 & 0.000 & 5.860 & 9.047 \\
\hline & TCAV-E\&M & 4 & Green & 1.00 & 0.796 & 1.160 & 0.000 & 5.649 & 10.568 \\
\hline & TIG-Bayesian & 3 & Green & 0.75 & 0.178 & 0.325 & 0.000 & 5.812 & 8.098 \\
\hline & RV-Bayesian & 3 & Green & 0.75 & 0.187 & 0.429 & 0.000 & 6.021 & 8.448 \\
\hline & RV-E\&M & 5 & Green & 1.25 & 0.606 & 1.216 & 0.000 & 5.240 & 9.985 \\
\hline & TRV-Bayesian & 3 & Green & 0.75 & 0.248 & 0.436 & 0.000 & 5.809 & 8.336 \\
\hline & TRIG-Bayesian & 3 & Green & 0.75 & 0.251 & 0.340 & 0.000 & 5.749 & 8.263 \\
\hline
\end{tabular}


Table 10: VaR prediction performance: using 17 model specifications and the 400 forecasts for the exchange returns

\begin{tabular}{|c|c|c|c|c|c|c|}
\hline \multicolumn{2}{|l|}{$\alpha=5 \%$} & \multirow{2}{*}{\multicolumn{2}{|c|}{ Violations VRate $/ \alpha$}} & \multicolumn{2}{|c|}{$\mathrm{AD}$} & \multirow{2}{*}{$\begin{array}{l}\text { Quantile criterion } \\
\text { function }\end{array}$} \\
\hline & & & & Mean & Max & \\
\hline \multirow[t]{17}{*}{ Euro vs US } & ST & 28 & 1.400 & 0.308 & 0.917 & 30.873 \\
\hline & $\mathrm{LT}$ & 22 & 1.100 & 0.307 & 1.214 & 29.164 \\
\hline & RiskMetrics & 22 & 1.100 & 0.278 & 0.964 & 28.226 \\
\hline & GARCH-n & 22 & 1.100 & 0.269 & 1.016 & 28.271 \\
\hline & GARCH-t & 24 & 1.200 & 0.272 & 1.035 & 28.455 \\
\hline & SAV-Bayesian & 19 & 0.950 & 0.284 & 0.771 & 28.154 \\
\hline & SAV-E\&M & 19 & 0.950 & 0.284 & 0.783 & 28.118 \\
\hline & AS-Bayesian & 20 & 1.000 & 0.272 & 0.759 & 28.254 \\
\hline & AS-E\&M & 20 & 1.000 & 0.273 & 0.775 & 28.241 \\
\hline & IG-Bayesian & 24 & 1.200 & 0.265 & 0.888 & 28.302 \\
\hline & TCAV-Bayesian & 18 & 0.90 & 0.309 & 0.822 & 28.483 \\
\hline & TCAV-E\&M & 16 & 0.800 & 0.317 & 0.791 & 28.363 \\
\hline & TIG-Bayesian & 22 & 1.100 & 0.267 & 0.818 & 27.910 \\
\hline & RV-Bayesian & 22 & 1.10 & 0.264 & 0.888 & 28.017 \\
\hline & RV-E\&M & 15 & 0.750 & 0.266 & 0.772 & 28.596 \\
\hline & TRV-Bayesian & 19 & 0.950 & 0.313 & 0.864 & 28.396 \\
\hline & TRIG-Bayesian & 21 & 1.050 & 0.284 & 0.938 & 28.454 \\
\hline \multirow[t]{17}{*}{ JP vs US } & ST & 24 & 1.200 & 0.336 & 1.063 & 30.350 \\
\hline & $\mathrm{LT}$ & 20 & 1.000 & 0.287 & 1.219 & 27.933 \\
\hline & RiskMetrics & 23 & 1.150 & 0.338 & 1.473 & 28.957 \\
\hline & GARCH-n & 19 & 0.950 & 0.343 & 1.114 & 28.362 \\
\hline & GARCH-t & 19 & 0.950 & 0.361 & 1.155 & 28.187 \\
\hline & SAV-Bayesian & 19 & 0.950 & 0.340 & 1.078 & 28.413 \\
\hline & SAV-E\&M & 19 & 0.950 & 0.335 & 1.088 & 28.378 \\
\hline & AS-Bayesian & 18 & 0.900 & 0.329 & 1.200 & 28.855 \\
\hline & AS-E\&M & 17 & 0.850 & 0.345 & 1.235 & 28.818 \\
\hline & IG-Bayesian & 19 & 0.950 & 0.332 & 1.123 & 28.068 \\
\hline & TCAV-Bayesian & 19 & 0.950 & 0.330 & 1.217 & 29.462 \\
\hline & TCAV-E\&M & 23 & 1.150 & 0.288 & 1.145 & 28.908 \\
\hline & TIG-Bayesian & 19 & 0.95 & 0.295 & 1.169 & 28.403 \\
\hline & RV-Bayesian & 19 & 0.95 & 0.332 & 1.138 & 28.412 \\
\hline & RV-E\&M & 18 & 0.900 & 0.319 & 1.021 & 28.447 \\
\hline & TRV-Bayesian & 18 & 0.900 & 0.341 & 1.307 & 29.142 \\
\hline & TRIG-Bayesian & 20 & 1.000 & 0.337 & 1.255 & 29.324 \\
\hline
\end{tabular}


Table 11: Counts of model rejections for the three quantile tests across each market, the portfolio and two exchange rate series

\begin{tabular}{|c|c|c|c|c|c|c|c|c|}
\hline \multirow[t]{2}{*}{ Specification } & \multicolumn{3}{|c|}{$1 \%$} & & \multicolumn{3}{|c|}{$5 \%$} & \multirow[b]{2}{*}{ total } \\
\hline & $L R_{u c}$ & $L R_{c c}$ & $D Q$ & total & $L R_{u c}$ & $L R_{c c}$ & $D Q$ & \\
\hline $\mathrm{ST}$ & 8 & 8 & 8 & 8 & 6 & 7 & 8 & 8 \\
\hline $\mathrm{LT}$ & 5 & 5 & 7 & 7 & 0 & 0 & 6 & 6 \\
\hline RiskMetrics & 4 & 4 & 5 & 5 & 1 & 0 & 5 & 5 \\
\hline GARCH-n & 4 & 4 & 5 & 5 & 1 & 1 & 5 & 5 \\
\hline GARCH-t & 1 & 0 & 5 & 5 & 2 & 1 & 6 & 6 \\
\hline SAV-Bayesian & 1 & 0 & 3 & 3 & 0 & 0 & 4 & 4 \\
\hline SAV-E\&M & 4 & 3 & 5 & 5 & 2 & 1 & 5 & 5 \\
\hline AS-Bayesian & 1 & 1 & 3 & 3 & 0 & 0 & 5 & 5 \\
\hline AS-E\&M & 5 & 5 & 5 & 5 & 3 & 1 & 6 & 6 \\
\hline IG-Bayesian & 0 & 0 & 1 & 1 & 0 & 0 & 2 & 2 \\
\hline IG-E\&M & 2 & 1 & 4 & 4 & 0 & 1 & 4 & 4 \\
\hline TCAV-Bayesian & 4 & 2 & 4 & 4 & 0 & 0 & 4 & 4 \\
\hline TIG-Bayesian & 2 & 1 & 4 & 4 & 0 & 0 & 5 & 5 \\
\hline RV-Bayesian & 0 & 0 & 1 & 1 & 2 & 0 & 2 & 2 \\
\hline RV-E\&M & 2 & 1 & 4 & 4 & 2 & 2 & 4 & 4 \\
\hline TRV-Bayesian & 1 & 0 & 4 & 4 & 1 & 0 & 3 & 3 \\
\hline TRIG-Bayesian & 1 & 1 & 3 & 3 & 2 & 0 & 4 & 4 \\
\hline
\end{tabular}

Table 12: Summary statistics for the 1\% VaR forecast criteria in Tables 4 and 5

\begin{tabular}{|c|c|c|c|c|c|c|c|c|c|c|c|}
\hline \multirow[b]{2}{*}{ Model } & \multicolumn{3}{|c|}{ VRate $/ \alpha$} & \multicolumn{2}{|c|}{ AD mean } & \multicolumn{2}{|c|}{$\mathrm{AD} \max$} & \multicolumn{2}{|c|}{ MRC } & \multicolumn{2}{|c|}{ Quantile criterion } \\
\hline & Mean & Median & RMSD & Mean & Median & Mean & Median & Mean & Median & Mean & Median \\
\hline ST & 4.656 & 4.710 & 3.286 & 0.773 & 0.878 & 2.951 & 2.834 & 12.788 & 14.907 & 29.599 & 34.192 \\
\hline LT & 1.974 & 2.330 & 1.160 & 1.146 & 1.136 & 2.989 & 3.078 & 14.295 & 16.442 & 28.350 & 31.432 \\
\hline RiskMetrics & 2.111 & 2.110 & 1.256 & 0.794 & 0.660 & 2.302 & 2.110 & 13.062 & 14.972 & 23.347 & 27.040 \\
\hline GARCH-n & 1.954 & 2.000 & 1.170 & 0.799 & 0.653 & 2.152 & 2.071 & 12.688 & 14.421 & 22.712 & 26.778 \\
\hline GARCH-t & 1.406 & 1.445 & 0.754 & 0.805 & 0.660 & 1.865 & 1.638 & 12.904 & 14.423 & 22.093 & 25.512 \\
\hline SAV-Bayesian & 1.496 & 1.555 & 0.677 & 0.628 & 0.550 & 1.661 & 1.196 & 12.792 & 14.620 & 21.858 & 24.872 \\
\hline SAV-E\&M & 2.053 & 1.890 & 1.308 & 0.712 & 0.745 & 2.157 & 1.875 & 13.048 & 14.654 & 23.128 & 25.602 \\
\hline AS-Bayesian & 1.549 & 1.765 & 0.810 & 0.735 & 0.675 & 1.978 & 1.835 & 12.554 & 14.488 & 22.128 & 24.531 \\
\hline AS-E\&M & 2.239 & 2.890 & 1.612 & 0.763 & 0.858 & 2.290 & 2.299 & 12.931 & 14.669 & 24.088 & 26.410 \\
\hline IG-Bayesian & 1.473 & 1.530 & 0.574 & 0.607 & 0.508 & \begin{tabular}{|l|}
1.714 \\
\end{tabular} & 1.509 & 12.436 & 14.395 & \begin{tabular}{|l|}
21.283 \\
\end{tabular} & 24.996 \\
\hline IG-E\&M & 1.603 & 1.540 & 0.793 & \begin{tabular}{|l|}
0.622 \\
\end{tabular} & 0.584 & 1.877 & 1.717 & 12.861 & 14.799 & 21.631 & 24.987 \\
\hline TCAV-Bayesian & 1.829 & 1.985 & 0.973 & 0.758 & 0.852 & 2.046 & 1.516 & 12.924 & 15.412 & 22.871 & \begin{tabular}{|l|}
24.369 \\
\end{tabular} \\
\hline TIG-Bayesian & 1.753 & 1.890 & 0.848 & 0.671 & 0.669 & \begin{tabular}{|l|}
1.780 \\
\end{tabular} & 1.609 & 12.734 & 14.802 & 22.128 & 25.644 \\
\hline RV-Bayesian & 1.269 & 1.225 & 0.601 & 0.739 & 0.794 & 1.954 & 1.578 & 12.469 & 14.277 & 21.321 & 25.078 \\
\hline RV-E\&M & 1.656 & 1.375 & 1.148 & 0.782 & 0.740 & 2.324 & 2.162 & 12.131 & 13.852 & 22.118 & 25.298 \\
\hline TRV-Bayesian & 1.430 & 1.415 & 0.664 & 0.738 & 0.757 & 2.044 & 1.885 & 12.702 & 14.570 & 22.028 & 26.653 \\
\hline TRIG-Bayesian & 1.378 & 1.330 & 0.689 & 0.863 & 0.967 & 2.170 & 2.021 & 12.741 & 14.676 & 22.435 & 26.815 \\
\hline
\end{tabular}


Table 13: Summary statistics for the $5 \%$ VaR forecast criteria in Tables 4 and 5

\begin{tabular}{|c|c|c|c|c|c|c|c|c|c|}
\hline \multirow[b]{2}{*}{ Model } & \multicolumn{3}{|c|}{ VRate $/ \alpha$} & \multicolumn{2}{|c|}{ AD mean } & \multicolumn{2}{|c|}{$\mathrm{AD} \max$} & \multicolumn{2}{|c|}{ Quantile criterion } \\
\hline & Mean & Median & RMSD & Mean & Median & Mean & Median & Mean & Median \\
\hline ST & 1.696 & 1.755 & 0.791 & 0.802 & 0.868 & 3.331 & 3.529 & 83.535 & 101.520 \\
\hline LT & 1.066 & 1.075 & 0.138 & 1.298 & 1.467 & 4.976 & 5.209 & 89.503 & 104.950 \\
\hline RiskMetrics & 1.244 & 1.29 & 0.307 & 0.843 & 0.900 & 3.726 & 3.996 & 80.308 & 96.915 \\
\hline GARCH-n & 1.248 & 1.24 & 0.327 & 0.873 & 0.974 & 3.590 & 3.747 & 80.341 & 97.492 \\
\hline GARCH-t & 1.306 & 1.31 & 0.384 & 0.868 & 0.968 & 3.694 & 3.907 & 80.487 & 97.563 \\
\hline SAV-Bayesian & 1.168 & 1.2 & 0.241 & 0.827 & 0.913 & 3.377 & 3.627 & 80.803 & 97.653 \\
\hline SAV-E\&M & 1.224 & 1.285 & 0.336 & 0.887 & 0.997 & 3.611 & 3.827 & 80.669 & 97.541 \\
\hline AS-Bayesian & 1.215 & 1.245 & 0.291 & 0.814 & 0.907 & 3.140 & 2.865 & 79.225 & 97.340 \\
\hline AS-E\&M & 1.274 & 1.32 & 0.429 & 0.859 & 0.923 & 3.486 & 3.786 & 80.108 & 97.864 \\
\hline IG-Bayesian & 1.185 & 1.2 & 0.241 & 0.816 & 0.889 & 3.316 & 3.318 & 80.335 & 97.826 \\
\hline IG-E\&M & 1.166 & 1.18 & 0.258 & 0.864 & 0.987 & 3.483 & 3.393 & 80.024 & 97.234 \\
\hline TCAV-Bayesian & 1.180 & 1.22 & 0.256 & 0.801 & 0.868 & 2.976 & 2.874 & 79.121 & 97.403 \\
\hline TIG-Bayesian & 1.181 & 1.2 & 0.249 & 0.796 & 0.862 & 3.046 & 3.181 & 79.260 & 97.353 \\
\hline RV-Bayesian & \begin{tabular}{|l|}
1.139 \\
\end{tabular} & 1.06 & 0.258 & \begin{tabular}{|l|}
0.767 \\
\end{tabular} & 0.896 & 3.408 & 3.482 & 77.814 & 94.308 \\
\hline RV-E\&M & 1.173 & 1.085 & 0.406 & 0.846 & 0.945 & 3.635 & 3.951 & 78.904 & 94.397 \\
\hline TRV-Bayesian & \begin{tabular}{|l|}
1.124 \\
\end{tabular} & 1.085 & 0.242 & \begin{tabular}{|l|}
0.769 \\
\end{tabular} & 0.810 & 3.180 & 2.795 & 78.927 & 95.640 \\
\hline TRIG-Bayesian & 1.194 & 1.115 & 0.286 & \begin{tabular}{|l|}
0.760 \\
\end{tabular} & 0.812 & 3.555 & 3.584 & 78.876 & 95.402 \\
\hline
\end{tabular}



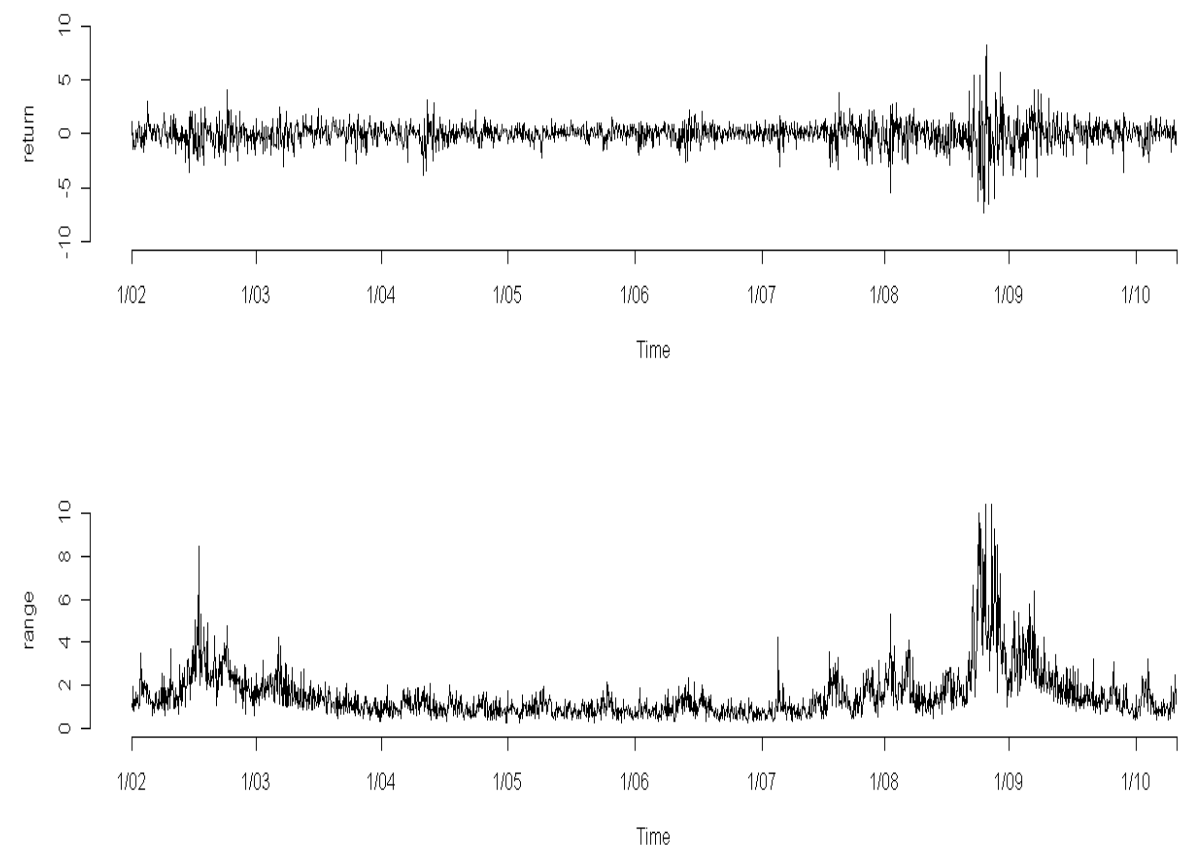

Figure 1: Time series plots for (a) portfolio's returns, (b) S\&P500 range.

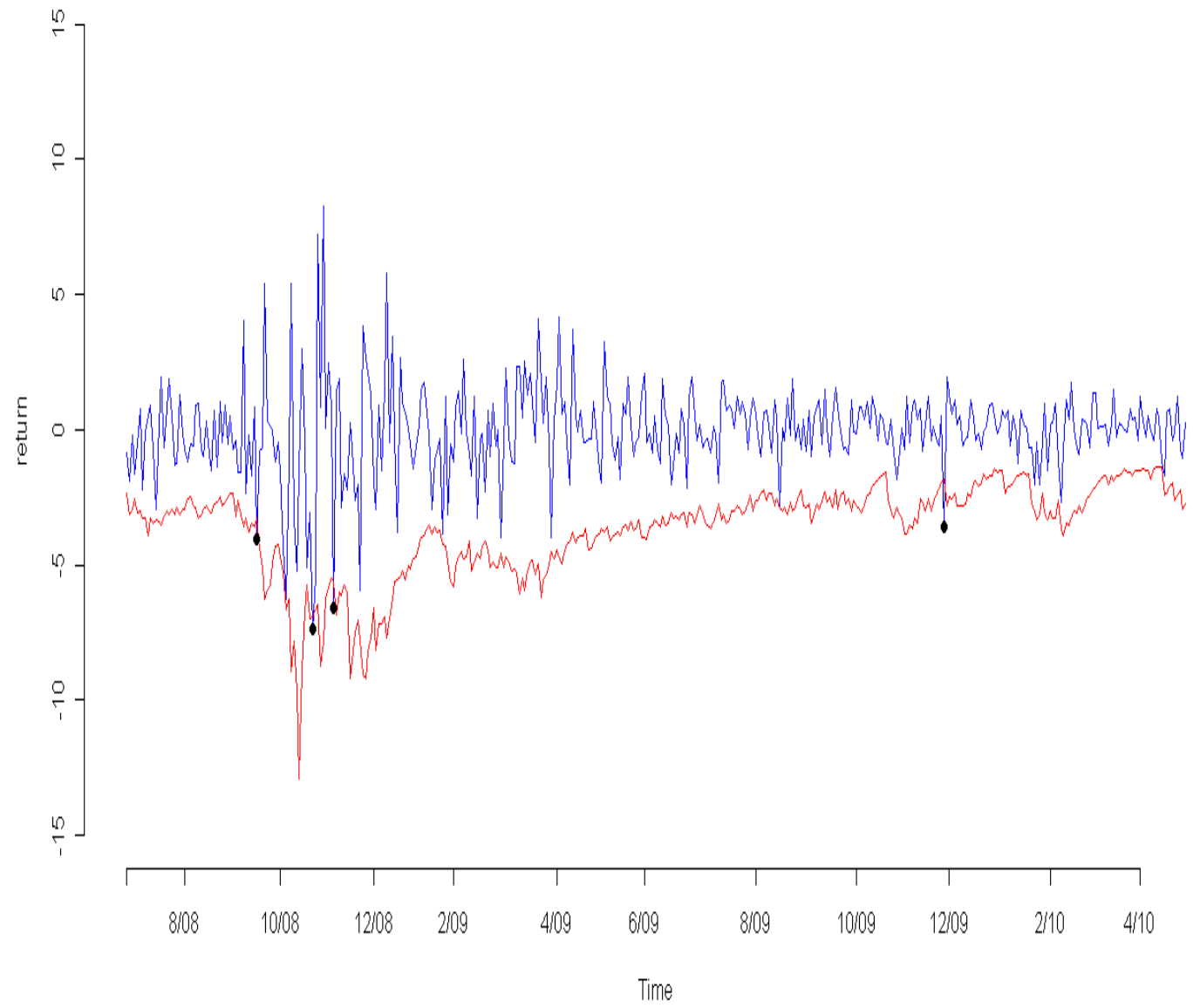

Figure 2: VaR forecasts of the TRIG model at the $1 \%$ level. 